\title{
A metabarcoding framework for facilitated survey of endolithic phototrophs with tufA
}

\author{
Thomas Sauvage ${ }^{1 *}$, William E. Schmidt ${ }^{1}$, Shoichiro Suda ${ }^{2}$ and Suzanne Fredericq ${ }^{1}$
}

\begin{abstract}
Background: In spite of their ecological importance as primary producers and microbioeroders of marine calcium carbonate $\left(\mathrm{CaCO}_{3}\right)$ substrata, endolithic phototrophs spanning both prokaryotic (the cyanobacteria) and eukaryotic algae lack established molecular resources for their facilitated survey with high throughput sequencing. Here, the development of a metabarcoding framework for the elongation factor EF-Ttu (tufA) was tested on four Illuminasequenced marine $\mathrm{CaCO}_{3}$ microfloras for the characterization of their endolithic phototrophs, especially the abundant bioeroding Ostreobium spp. (Ulvophyceae). The framework consists of novel tufA degenerate primers and a comprehensive database enabling Operational Taxonomic Unit (OTU) identification at multiple taxonomic ranks with percent identity thresholds determined herein.
\end{abstract}

Results: The newly established tufA database comprises 4057 non-redundant sequences (from 1339 eukaryotic and prokaryotic phototrophs, and 2718 prokaryotic heterotrophs) including 27 classes in 10 phyla of phototrophic diversity summarized from data mining on GenBank ${ }^{\circledR}$, our barcoding of $>150$ clones produced from coral reef microfloras, and $>300$ eukaryotic phototrophs (>230 Ulvophyceae including $>100^{\prime}$ Ostreobium'spp., and $>70$ Florideophyceae, Phaeophyceae and miscellaneous taxa). Illumina metabarcoding with the newly designed primers resulted in 802 robust OTUs including 618 phototrophs and 184 heterotrophs (77 and $23 \%$ of OTUs, respectively). Phototrophic OTUs belonged to 14 classes of phototrophs found in seven phyla, and represented $\sim 98 \%$ of all reads. The phylogenetic profiles of coral reef microfloras showed few OTUs in large abundance (proportion of reads) for the Chlorophyta (Ulvophyceae, i.e. Ostreobium and Phaeophila), the Rhodophyta (Florideophyceae) and Haptophyta (Coccolithophyceae), and a large diversity (richness) of OTUs in lower abundance for the Cyanophyta (Cyanophyceae) and the Ochrophyta (the diatoms, 'Bacillariophyta'). The bioerosive 'Ostreobium' spp. represented four families in a large clade of subordinal divergence, i.e. the Ostreobidineae, and a fifth, phylogenetically remote family in the suborder Halimedineae (provisionally assigned as the 'Pseudostreobiaceae'). Together they harbor 85-95 delimited cryptic species of endolithic microsiphons.

Conclusions: The novel degenerate primers allowed for amplification of endolithic phototrophs across a wide phylogenetic breadth as well as their recovery in very large proportions of reads (overall $98 \%$ ) and diversity (overall $77 \%$ of OTUs). The established companion tufA database and determined identity thresholds allow for OTU identification at multiple taxonomic ranks to facilitate the monitoring of phototrophic assemblages via metabarcoding, especially endolithic communities rich in bioeroding Ulvophyceae, such as those harboring 'Ostreobium' spp., Phaeophila spp. and associated algal diversity.

\footnotetext{
*Correspondence: tomsauv@gmail.com

1 Department of Biology, University of Louisiana at Lafayette, Lafayette, LA

70503, USA

Full list of author information is available at the end of the article
} 
Keywords: Algae, Amplicon Metagenomics, Bryopsidales, Calcium carbonate, Coral reef, Illumina MiSeq, Metabarcoding, Next generation sequencing, Ostreobium, Phaeophila, Phototrophs, Plastid, Rhodoliths, tufA, Ulvophyceae

\section{Background}

Endolithic phototrophs are major primary producers [68] and microbioeroders in marine carbonate substrata [98]. They may colonize the surface and cavities of the substratum (chaesmoendoliths and cryptoendoliths, respectively), as well as actively penetrate it (euendoliths) wherever sufficient light penetrates for photosynthesis. They may be found in aragonite, calcite, or a combination of both, in e.g. live and dead corals, mollusk shells, and crustose coralline algae (CCA) [97]. In calcium carbonate $\mathrm{CaCO}_{3}$-building ecosystems, such as CCA ridges, coral reefs, oyster reefs and rhodolith beds, euendolithic phototrophs play a critical role in the dynamic balance between constructive (accretion) and destructive (dissolution) processes [36]. With upcoming global changes, this balance may be negatively affected by enhancing the bioerosive power of boring phototrophs, as measured under projected ocean acidification [higher partial pressure of carbon dioxide $\left.\left(\mathrm{pCO}_{2}\right)\right]$ and increased temperature regimes $[81,82,99]$. The prospect of accelerated biogenic $\mathrm{CaCO}_{3}$ dissolution poses strong concerns for the future maintenance of the structural integrity and functionality of these ecosystems considering the biodiverse assemblages of micro- and macro-organisms they support [72, 73].

Commonly reported euendolithic phototrophs include the eukaryotic green algal genera Ostreobium Bornet and Flahault (order Bryopsidales) and Phaeophila Hauck ('Ulvales-Ulothrichales') (both in class Ulvophyceae, phylum Chlorophyta), and prokaryotic (eubacterial) blue-green algal genera (all in class Cyanophyceae, phylum Cyanophyta) such as Mastigocoleus Lagerheim ex Bornet and Flahault, and Plectonema Thuret ex Gomont [98]. They also include microscopic alternate life stages of otherwise conspicuous alga, e.g. the Conchocelis-stage of the red alga Porphyra C. Agardh (Bangiophyceae, Rhodophyta) and endolithic vegetative networks underlying diminutive epilithic Bryopsidales, e.g. Pseudochlorodesmis Børgesen and Caulerpa ambigua Okamura $[1,50]$. Among the above, Ostreobium microsiphons are omnipresent agents of bioerosion [98], although the microfilaments of the genus Phaeophila are also often reported (e.g. see [12, 79]). The molecular diversity of Ostreobium spp. remains particularly unexplored toward establishing comprehensive sequence reference databases for the profiling of endolithic communities via metabacording (e.g. [14]).

The skeleton of reef-building scleractian coral species (Cnidaria) is abundantly colonized by Ostreobium [54, 60, 61] (Fig. 1), in which it develops a dense green layer underlying the animal tissue and where, as part of the coral holobiont, it may play a role as a nutritional ally (i.e. metabolite translocation, [31]). Previously, GutnerHoch and Fine [38] investigated the molecular diversity of this 'green layer' with $r b c \mathrm{~L}$ in two coral species from the Red Sea in order to gain insights into potential patterns of association of Ostreobium haplotypes with coral species. While these authors reported some possible haplotype-to-coral species distributional patterns, they also revealed multiple $r b c \mathrm{~L}$ haplotypes of Ostreobium,

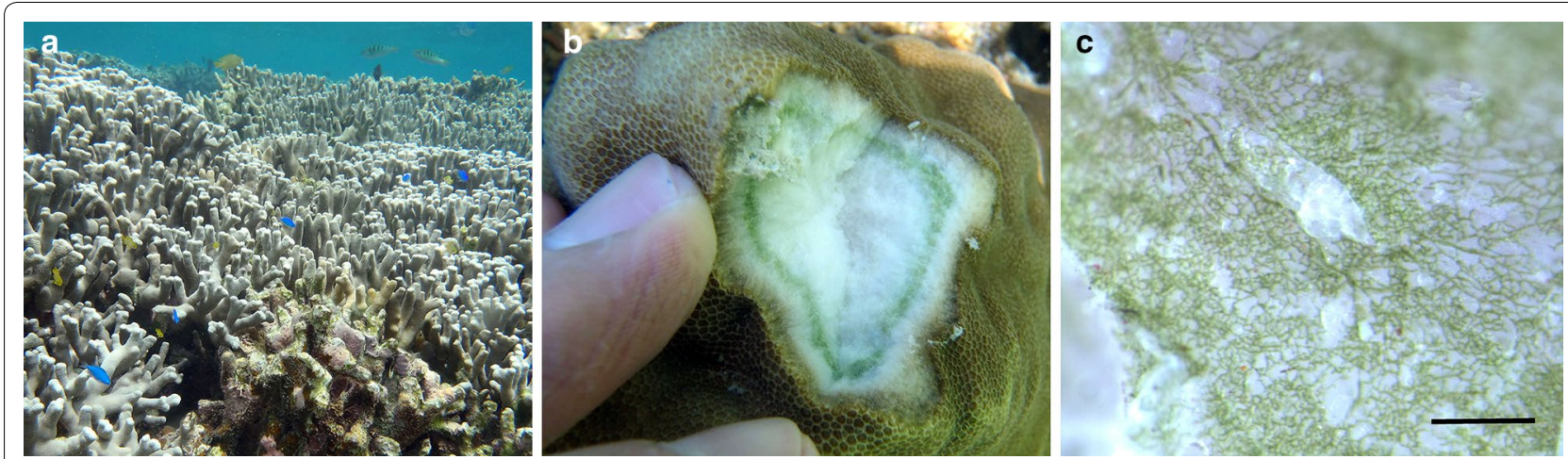

Fig. 1 Ostreobium in situ in the Ryukus. a Coral reef habitat where microfloras harboring Ostreobium spp. underlay live coral tissue (picture background) and limestone often covered by epilithic turf algae and crustose coralline algae (foreground). b Fragmented coral colony showing Ostreobium's 'green layer' found below live coral tissue, here Porites sp. c Calcium carbonate colonized by Ostreobium sp. microsiphons (Scale bar approximately $20 \mu \mathrm{m})$ 
whose taxonomic breadth remains unknown (with regard to species diversity and the taxonomic rank attributed to this novel diversity) from a lack of phylogenetic context in their analysis. Earlier, Verbruggen et al. [102] suggested that the genus Ostreobium might actually represent an entire suborder of microsiphonous species that they informally proposed as the 'Ostreobidineae' (next to two other informally accepted suborders in the Bryopsidales, the Bryopsidineae and Halimedineae, see [41]) based on the early branching of a single Ostreobium specimen in a comprehensive multi-marker phylogeny of the Bryopsidales [Chloroplast $16 \mathrm{~S}$ ribosomal DNA (rDNA), RuBisCO Large subunit $(r b c \mathrm{~L})$, and elongation EF-Tu (tufA)]; however, this remains to be substantiated. Overall, considering the high density of Ostreobium microsiphons in the so-called coral 'green layer', this microhabitat is particularly convenient to target in order to rapidly build reference barcode data sets.

Metabarcoding represents a novel terminology [96] for amplicon-based metagenomics (e.g. usually targeting $16 \mathrm{~S},[18])$ in contrast to whole-genome metagenomics [65]. Important limitations to metabarcoding include the availability of universal primers amplifying the targeted diversity with minimal taxon bias [51, 59], the clustering of next-generation reads into biologically relevant Operational Taxonomic Unit (OTU) through the elimination of sequencing error/noise (e.g. [25, 26, 88]), and building up taxonomy-curated reference sequence database for OTU identification/annotation (e.g. Greengenes, [21]; Ribosomal Database Project [RDP], [13]; silva, [77]). Currently, a metabarcoding framework specifically developed to target lower phototrophs (i.e. the algae sensu lato: prokaryotic blue-green algae and eukaryotic algae) and their recovery in large proportions of reads is inexistent and is sorely needed to facilitate the monitoring of endolithic phototrophs and other microbial/algal assemblages. A recent progress toward metabarcoding phototrophs was made with the establishment of a curated chloroplast $16 \mathrm{~S}$ rDNA database (primarily for phytoplankton taxa, PHYTO-REF, [17]); however, primers used to amplify $16 \mathrm{~S}$ are generally highly conserved in prokaryotes and tend to recover low proportions of phototrophic organisms from environmental mixtures where microbial DNA from heterotrophic phyla is inherently overdominant. For instance, in a study of freshwater phytoplankton communities with 16S (V3-V4 region), only $9 \%$ of reads represented phototrophic organisms [27]. Likewise, 16S libraries sequenced from coral tissue and their underlying endolithic communities enumerated very few phototrophs in comparison to heterotrophs (e.g. see [59, 85, 94]).

A candidate DNA marker for metabarcoding phototrophs is the gene encoding the protein chain elongation factor EF-Tu, or tufA, whose role in the RNA translation machinery is deeply conserved among eubacteria and their eukaryotic endosymbiotic offshoot, the organelles [52], especially in the chloroplast (translation mechanisms are modified in the mitochondrion, see [95]). Iwabe et al. [42] first used the elongation factors EF-Tu (and its homolog in archaea and eukaryotic nucleus, the elongation factor 1 alpha, EF-1a, [40]) to examine deep (domain) phylogenetic relationships (EF-Tu and EF-1a show some amino acid conservation but their DNA sequences are highly divergent). Later, Delwiche et al. [19] demonstrated the cyanobacterial (Cyanophyta) origin of all plastids in a single-gene phylogeny of tufA rooted with heterotrophs. Subsequently, tufA has gained much popularity in phylogenetic and systematic studies of diverse phototrophs (e.g. $[5,29,66,84,106])$ and was also recommended as a standard marker for the routine barcoding of the Chlorophyta [86] for its high amplification rate $(95 \%$, except in the Cladophorales, Ulvophyceae), and faster evolving rate relative to other commonly used markers in this phylum [e.g. $r b c \mathrm{~L}$ or rDNA markers such as the large subunit (LSU), 23S universal plastid amplicon (UPA) and the internal transcribed spacer (ITS)]. In early branching members of the Streptophyta (the Charophytes Mesostigma and Chlorokybus), tufA is chloroplast-encoded while in the remainder of this phylum (i.e. the higher phototrophs), it is nuclear-encoded [3]. In some eubacteria (Gram-negative), including some Cyanophyta, e.g. in the Oscillatoriales (e.g. Oscillatoria nigro-viridis PCC7112), tufA paralogs known as elongation factor EF-Tu 2 or tufB may exist [45, 53, 90]; however, these usually undergo concerted evolution and thus exhibit very low divergence [100]. Considering the universality of tufA and the large amount of data available on Genbank ${ }^{\circledR}$ for the order Bryopsidales $(>1300$ accessions), this marker is thus well-suited for the barcoding of Ostreobium spp. and metabarcoding of microfloras dominated by its microsiphons and associated algal diversity.

Here, we established a metabacording framework consisting of newly developed primers, a curated database of phototrophic diversity $\left(\mathrm{GenBank}^{\circledR}\right.$ data, a clone library of endolithic phototrophs, and new reference barcodes for Ostreobium spp. and related taxa), a provisional classification scheme for cryptic endoliths in the order Bryopsidales, and recommended identity thresholds for the taxonomic annotation of phototrophs at high levels (domain to class) and at lower levels in the Ulvophyceae (order to family, i.e. in the Bryopsidales and Ulvales). This framework, geared toward molecular ecology studies of endolithic phototroph assemblages rich in bioeroding Ulvophycean taxa (e.g. Ostreobium spp.), is tested on four Illumina-metabarcoded $\mathrm{CaCO}_{3}$ microfloras. 


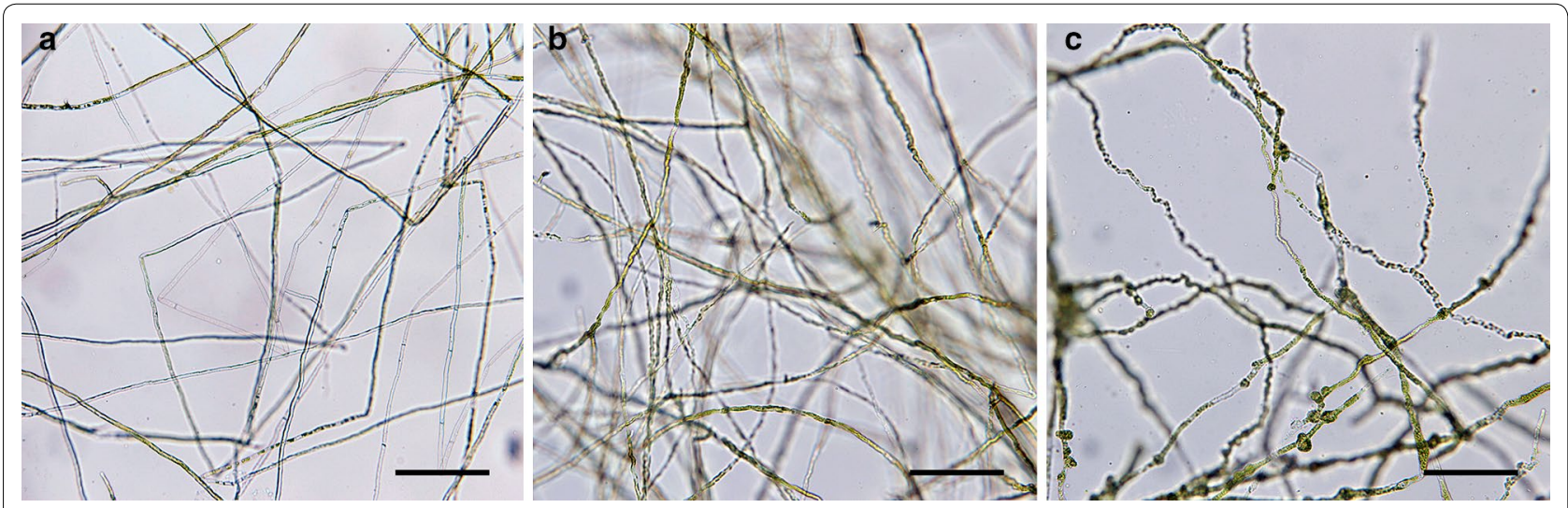

Fig. 2 Free-living Ostreobium in culture. a Specimen belonging in provisional family 'Hamidaceae' (TS1385, note that some chloroplast-depleted siphons may falsely appear septated), b 'Maedaceae' (TS1410B), and c'Odoaceae' (TS1408). No picture is available for the 'Unarizakiaceae'. Scale bar $50 \mu \mathrm{m}$

\section{Methods}

\section{Building up Ostreobium and reference tufA diversity}

Ostreobium specimens were sequenced primarily from the $\mathrm{CaCO}_{3}$ of densely colonized coral skeletons collected throughout the Ryukyu archipelago and culture starters established from them (Fig. 2, Additional file 1: Table S1). A few additional specimens originated from endolithic siphons underlying rhodolith-forming encrusting red algae from the Gulf of Mexico (GM) and also from starters established from oyster shells collected in Florida and corals from miscellaneous localities (Additional file 1: Table S1). For culturing, colonized blocks of $\mathrm{CaCO}_{3}$ were subsetted to $\sim 0.04-0.25 \mathrm{~cm}^{2}$ and incubated for $10-20$ days in $60 \mathrm{~mL}$ polypropylene cups (Diamond $\mathrm{d}^{\mathrm{TM}}$ ) with 20-30 mL half strength Provasoli enriched seawater medium [75] supplemented with $1.25 \mathrm{mg} / \mathrm{L}$ germanium dioxide $\left(\mathrm{GeO}_{2}\right)$ under light-dark 14:10 cycles $\left(50 \mu \mathrm{mol} \mathrm{m}{ }^{-2} \mathrm{~s}^{-1}\right)$ and at room temperature $\left(22 \pm 1{ }^{\circ} \mathrm{C}\right)$. Specimens were extracted with a DNeasy Plant Mini Kit (Qiagen, Valencia, CA, USA) and tufA amplified by polymerase chain reaction (PCR) as previously published $[29,39,87]$ and/or with novel primers in various combination (Table 1). Successful PCR products were Sanger-sequenced commercially and chromatograms assembled in Sequencher v.5.1 (Gene Codes, Ann Arbor, Michigan, USA). To further increase reference sequence context, tufA barcodes were also generated for macroscopic ( $>5-20 \mathrm{~cm}$, e.g. Codium, Halimeda, Rhipilia) and diminutive members of the Bryopsidales $(<2 \mathrm{~cm})$ (e.g. the polyphyletic 'Pseudochlorodesmis' species complex, [103]; and the monophyletic Caulerpa 'ambigua' species complex, [23]). Likewise, miscellaneous 'Ulvales-Ulothrichales' that occasionally emerged in cultures (Additional file 1: Table S1) as well as several Florideophyceae (Rhodophyta) and few Phaeophyceae (Ochrophyta)
Table 1 Newly designed PCR primers for tufA barcoding and metabarcoding

\begin{tabular}{lllll}
\hline Name & Sequence $\left(\mathbf{5}^{\prime} \mathbf{- 3}^{\prime}\right)$ & bp & $\mathbf{G C}(\%)$ & $\mathbf{T m}\left({ }^{\circ} \mathbf{C}\right)$ \\
\hline tu470F & TTTTAATGGCTGTCGAAAATGTTG & 24 & 33.3 & 52.8 \\
tubryoF & GCAGATGGTCCAATGCCWCAAAC & 23 & 52.2 & 59 \\
tubryoR & CCWGGTTTAGCTAAAACCATNCC & 23 & 45.7 & 54.9 \\
env_tufAF & TGGGTDGAHAADATTTWYNMNY- & 28 & 33.3 & $46.5-62.8$ \\
& TRATGR & & & \\
env_tufAR & TNACATCHGTWGTWCKNACATA- & 28 & 35.1 & $49.8-60.6$ \\
& RAAYTG & & & \\
\hline
\end{tabular}

a Metabarcoding primers

maintained in the algal collections at the University of Louisiana at Lafayette (LAF) were also barcoded (Additional file 2: Table S2). Corals and reef substratum collections in the Ryukyu archipelago were conducted under Permit 24-3 delivered by the Okinawa Prefecture Fishery Control 33-2-40. Collections in Florida's coastal waters were permitted by a 'Saltwater fishing' license from the Fish and Wildlife (\#1000427446) and those from Garden Key, Dry Tortugas by the U.S. National Park Service (\#DRTO-2013-SCI-0015).

\section{Microflora samples}

Several microflora specimens were selected for environmental sequencing to build a clone library and for metabarcoding (Table 2, Fig. 3). These consisted of limestone fragments from reef rubble or substratum adjacent to coral colonies originating from the Ryukyu archipelago (JP01, JP03, JP04, JP06, JP07 and JP25), the Florida Keys (FL01 and FL02) and a northwestern Gulf of Mexico rhodolith (GM14). These specimens were lightly drilled within $\mathrm{CaCO}_{3}$ patches devoid of crustose 
Table 2 Limestone samples processed via cloning and metabarcoding

\begin{tabular}{llllll}
\hline Sample & Clon./Metab. $^{\text {a }}$ & Site & Depth $(\mathbf{m})$ & Date & Substratum \\
\hline FL01 & - / & Big pine key, Florida & 4 & $09 / 2013$ & Reef rubble \\
FL02 & $-/+$ & Big pine key, Florida & 4 & $09 / 2013$ & Reef rubble \\
JP01 & $+/-$ & Ryukyus & & Reef rubble \\
JP03 & $+/-$ & Ryukyus & $<5$ & $07 / 2012$ & Reef rubble \\
JP04 & $+/-$ & Ryukyus & $<5$ & $07 / 2012$ & Reef rubble \\
JP06 & $+/-$ & Ryukyus & $<5$ & $07 / 2012$ & Reef matrix \\
JP07 & $+/+$ & Ryukyus & $<5$ & $07 / 2012$ & Reef matrix/rubble \\
JP25 & $+/-$ & Ryukyus & $<5$ & $07 / 2012$ & Reef rubble \\
GM14 & $-/+$ & Ewing Bank, NWGM & $07 / 2012$ & Rhodolith \\
E09 & $+/-$ & - & 57 & $08 / 2008$ & Aquarium window \\
S15 & $+/-$ & NWGM & - & $65 / 2013$ & Lithophyllum sp. \\
\hline
\end{tabular}

a Cloning and/or metabarcoding: performed (+), not performed (-)

b Northwestern Gulf of Mexico

c Mixed locations and sampling dates within the archipelago

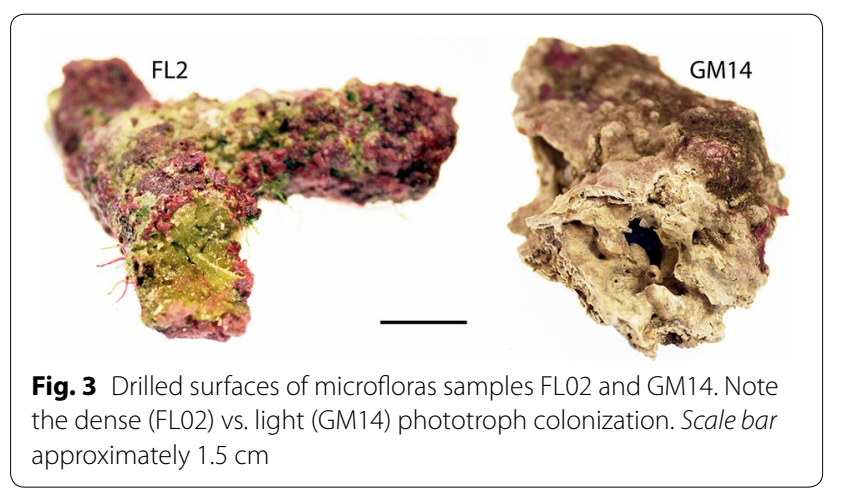

epiliths with a sterile $1.6 \mathrm{~mm}(1 / 16$ ") bit mounted on a Flex-Shaft Attachment (Model 225) powered by a Dremel 3000 rotary tool $\left(\right.$ Dremel $^{\circledR}$, Racine, WI, USA). For each microflora sample, DNA from a total of 40-80 mg of pulverized $\mathrm{CaCO}_{3}$ obtained from multiple drills was extracted with a PowerSoil DNA Isolation kit (MO BIO Laboratories, Carlsbad, CA, USA). Samples JP01 to JP07 were each extracted from limestone fragments pooled from multiple locations in the Ryukyus to maximize tufA sequence diversity. Four of the above microflora samples were metabarcoded (JP07, FL01, FL02 and GM14); samples JP07 and FL02 were densely colonized with endolithic taxa whereas samples FL01 and GM14 were more lightly colonized (Fig. 3) and were thus metabarcoded at different sequencing depths (see "tufA microflora assays" section). In preliminary cloning assays, two miscellaneous environmental samples comprising an aquarium window scrap exhibiting encrusting Ulvophyceae (E09), and endoliths underlaying a crustose coralline Lithophyllum sp. specimen (S15) were also processed (Table 2).

\section{tufA microflora assays}

Degenerate primers (env_tufAF/env_tufAR, see Table 1) were designed with HYDEN [58] on a phylogenetically diverse alignment of phototroph sequences. These primers target a 462 base pair (bp) amplicon ( 407 bp without incorporated primers) that is nested in the $3^{\prime}$ half of tufA (see Fig. 4, created with WebLogo 3.4, [15]). The produced amplicon does not overlap with the $5^{\prime}$ intron found in the Euglenophyta [67], and is devoid of codon insertion/deletions (some exist within the amplicon in heterotrophic bacteria). PCR products for cloning or Illumina-metabarcoding were amplified from DNA extracts on a low temperature/long annealing and long extension cycle to maximize diversity recovery $\left(3 \mathrm{~min}\right.$ at $95^{\circ} \mathrm{C}$, 1 min steps at 94,42 , and $72{ }^{\circ} \mathrm{C}$ for 40 cycles and a $5 \mathrm{~min}$ final $72{ }^{\circ} \mathrm{C}$ extension). Samples generally required $1: 10$ th to $1: 100$ th dilution for successful amplification due to DNA extract concentration variation and/or the potential presence of PCR inhibitors commonly found in algal samples (such as polysaccharides and natural products, [105]). For cloning, PCR products were separated with a TOPO ${ }^{\circledR}$ TA Cloning ${ }^{\circledR}$ Kit (One Shot ${ }^{\circledR}$ Top 10 chemically competent $E$. coli, Invitrogen ${ }^{\mathrm{TM}}$, Life Technologies, Grand Island, NY) following the manufacturer's protocol. Clones were grown on LB agar plates containing $50 \mu \mathrm{g} /$ $\mathrm{mL}$ Ampicillin and $40 \mu \mathrm{g} / \mathrm{mL}$ X-Gal. PCR was performed on white colonies with primers env_tufAF and M13R (M13 Reverse priming site on TOPO ${ }^{\circledR}$ vector) and conditions as above. Colony PCR products of correct size were sequenced commercially and assembled in Sequencher v5.1. For metabarcoding, DNA extracts were shipped to MRDNA (http://www.mrdnalab.com, Shallowater, TX, USA), where PCR products were amplified using a HotStarTaq Plus Master Mix Kit (Qiagen, Valencia, CA, 


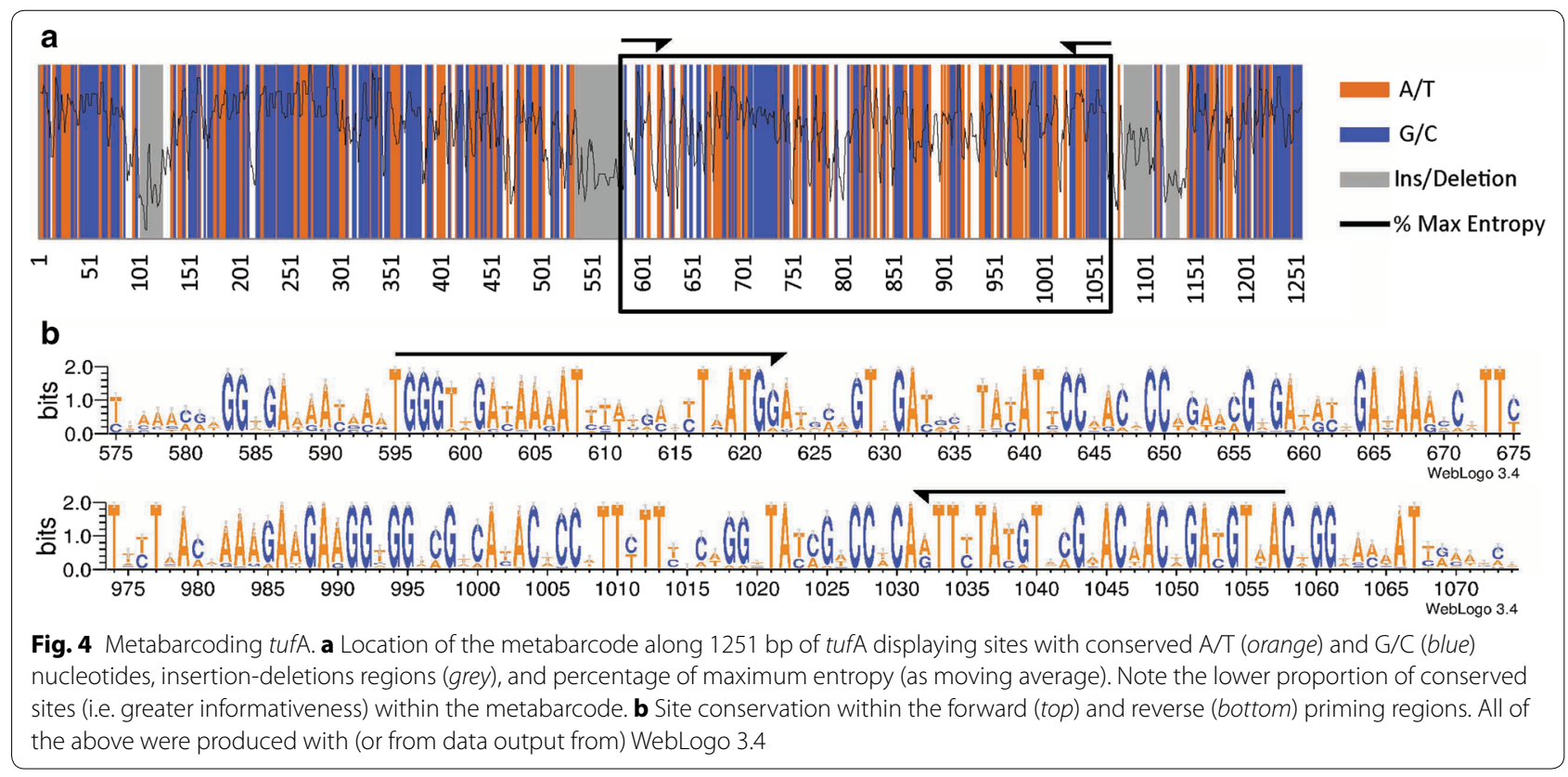

USA) with indexed env_tufAF primers (Additional file 3: Table S3). The amplification of the densely colonized samples JP07 and FL02 at MRDNA produced strong PCR products, while the more lightly colonized samples GM14 and FL01 resulted in weaker PCR products (in congruence with PCR testing conducted at LAF prior to the shipping of these samples). Thus, JP07 and FL02 products were pooled in higher proportion for deep sequencing while products from FL01 and GM14 were normalized and pooled with microbiota assays from other customers (i.e. 16S rDNA) to produce a nominal 20,000 reads per assay (as routinely performed at MRDNA). The prepared library (TruSeq DNA Sample Prep Kit for $2 \times 250$ bp paired-ends) was sequenced on the lane of an Illumina MiSeq Platform (Illumina Inc, San Diego, CA, USA).

\section{De novo clustering of tufA OTUs}

Raw Miseq reads were processed with the USEARCH pipeline (http://www.drive5.com/uparse/) [26] to overlap paired-ends (-fastq_mergepairs, $\mathrm{Q}=2$ ), demultiplexing (including stripping of indexes and forward primers, fastq_strip_barcode_relabel.py script), filter for high quality reads (-fastq_filter) of appropriate size (expected error $\mathrm{E}>0.5$, min $400 \mathrm{bp}$, max $440 \mathrm{bp}$ ), global trimming ( $5^{\prime}$ cropping of $20 \mathrm{bp}, 3^{\prime}$ cropping of $50 \mathrm{bp}$ ), and removal of noisy reads after dereplication (singletons, doubletons and tripletons found across the entire data set), as well as chimeras and contaminants (non-tufA reads) with UCHIME (command -uchime_ref) and UBLAST (-ublast) (Table 3). The dereplication file (with remaining
Table 3 Remaining reads throughout the UPARSE pipeline as counts and percentage of raw reads

\begin{tabular}{lcl}
\hline Raw & $\mathbf{4 , 9 1 7 , 8 8 8}$ & $\mathbf{1 0 0 \%}$ \\
\hline Paired-ends merging & $4,720,138$ & 96 \\
Demultiplexing & $1,928,898$ & 39 \\
Read quality filter $^{\text {Read size filter }}{ }^{\mathrm{a}}$ & $1,465,438$ & 30 \\
Denoising $^{\mathrm{b}}$ & $1,331,438$ & 27 \\
Decontamination $^{\mathrm{c}}$ & 837,069 & 17 \\
\hline
\end{tabular}

a Reads used for OTU mapping

b Singletons, doubletons and tripletons

c Non-tufA reads and chimeric reads

reads, average read length of $375 \mathrm{bp}$ ) was then clustered with UPARSE (-cluster_otus) at the recommended $97 \%$ global threshold and with SWARM [62] at multiple local thresholds ( $\mathrm{d}=1$ to 16$)$ to explore its clustering optima (none published). UPARSE (97 \%) and SWARM $(\mathrm{d}=10)$ outputs were then parsed to identify robust core OTUs generated by both algorithms to follow recent recommendations for reproducibility with the use of multiple algorithms [88]. Core OTUs were then mapped at the $97 \%$ level (with -usearch_global) against the quality-filtered (merged) reads (including singletons, doubletons and tripletons) to produce an OTU abundance table (uc2otutab.py script). Cumulative and non-cumulative rank-abundance curves were built from this table to explore the microflora's assemblage structure. 


\section{tufA reference database}

GenBank $^{\circledR}$ 's tufA sequences were summarized into a non-redundant local database augmented with newly generated Sanger data (including clones). We retrieved with BLASTn the 20,000 closest matches to multiple tufA sequences representing major branches of photosynthetic algal diversity (eukaryotic and prokaryotic). Search results were pooled, filtered for sequences $>300 \mathrm{bp}$, and dereplicated (100\% identity). Following preliminary tree building, sequences of prokaryotic heterotrophs (eubacteria other than cyanobacteria) were segregated and used for separate searches as above. The numerous resulting heterotroph sequences were dereplicated at $99 \%$. We excluded the non-photosynthetic apicoplast-encoded tufA of the Apicomplexa [11] and the nuclear-encoded tufA of the Streptophyta [3] because of conflicting phylogenetic signal (see $[19,43,49])$ and irrelevance to the target group/habitat investigated herein (i.e. lower phototrophs inhabiting $\mathrm{CaCO}_{3}$ ). Nonetheless, the deepest branch of the Streptophyta, i.e. the Charophyte algae Mesostigma and Chlorokybus [56, 101] were maintained in the database because their tufA is chloroplast-encoded. The only two sequences available for the terrestrial order Trentepohliales (Cephaleuros and Trentepohlia, Ulvophyceae) were also excluded for their large divergence with other eukaryotic tufA and ambiguous phylogenetic placement (until genome sequencing clarifies the organellar localization of their tufA, i.e. nuclear or chloroplastic). The final sorted tufA database comprised 4057 sequences (1339 phototrophs and 2718 heterotrophs). We followed PHYTO-REF for the classification of Diatoms (namely a single phylum-class noted as 'Bacillariophyta' within the Ochrophyta) and otherwise followed the Algaebase class-scheme [37]. Within the Ulvophyceae, we adopted the combined notation 'Ulvales-Ulothrichales' [55]. A summary of the database sequence content is provided in Additional file 4: Table S4.

\section{tufA phylogenies}

Phylogenetic reconstruction of the tufA database was performed to visualize its diversity and for clade-based (topological) identification of clones and OTU sequences $(\mathrm{n}=802)$. In order to produce a diverse heterotroph outgroup while greatly reducing sequence number for efficient computation time, we 'framed' bacterial reference diversity by selecting the most distant haplotypes in large clades observed in preliminary trees $(n=556$ heterotroph sequences kept). The final dataset comprising 2697 sequences was translated into amino acids and aligned with MUSCLE [24]. Regions with poor homology (codon insertion/deletions) were cropped between sites of conserved amino acids (see Fig. 4) and the sequences translated back to nucleic acids. The final alignment
(891 bp) was then ran with RAxML-HPC2 on the CIPRES computer cluster (http://www.phylo.org) with a GTR $+\mathrm{I}+\mathrm{G}$ model of evolution partitioned per codon position, 200 topological searches from random restarts and 1000 bootstrap replicates for node support estimation. To further detail diversity in euendolithic Ulvophyceae, and for species delimitation analyses (see below), the above phototroph alignment was subsetted for the orders Bryopsidales and 'Ulvales-Ulothrichales' and few outgroup taxa. Previously dereplicated barcode data were reintroduced to show sampling effort in these orders. The final alignment comprising 906 sequences was used for phylogenetic analysis as listed above (with 1000 restarts and 1000 bootstrap replicates) and species delimitation analyses (see below). All trees were edited in iTOL [57].

\section{Ulvophycean species delimitation}

Branch lengths were extracted from the RAxML Ulvophyceae tree with function cophenetic.phylo of the package APE in $R[69,78]$ to produce a distance matrix as input for the standalone version of the Automatic Barcode Gap Discovery software (ABGD, [76]). The latter was run with minimum (pmin) and maximum (pmax) intraspecific distance priors comprised between 0.001 and 1 in 100 steps, and with a 0.5 relative gap width. Alternative species boundaries hypotheses were produced with the general mixed yule coalescence (GMYC) model with the package SPLITS in R [35], with the single threshold method based on an ultrametric tree generated in BEAST v2.0 [8] using a relaxed log-normal clock with a constant population coalescent as prior, and a GTR $+\mathrm{I}+\mathrm{G}$ model of evolution partitioned per codon position. Markov Chain Monte Carlo (MCMC) chains were run for 30 million generations (sampled every 1000th generations) and the quality of the run assessed in Tracer v1.6 [80] to ensure that effective sample size (ESS) values were $>200$ with the default burnin (3000 trees).

\section{Divergence and identity thresholds}

The tufA database alignment was cropped to the metabarcode length (i.e. clustered OTUs of $375 \mathrm{bp}$ ) and a pairwise percent identity matrix created with function dist. dna in package APE (R) (a few sequences with $>20 \mathrm{bp}$ of missing data on their $5^{\prime}$ or $3^{\prime}$ side were excluded in order to avoid inflation of computed percent identity values). From this distance matrix, boxplots depicting the amount of divergence within individual families, suborders and orders of the Ulvophyceae ('Ulvales-Ulothrichales' and Bryopsidales) were drawn in order to examine their validity. Next, to define clade-based (conservative) identity thresholds for the rapid annotation of OTU at multiple taxonomic ranks, the function sppDist of the package SPIDER in R [10] was used to compute 
the overall intra- and inter- clade divergence values for all phototrophs at high-levels ranks (domain, phylum, and class clades) and at lower levels for the Ulvophyceae (order, suborder, and family clades, as well as molecular species clades delimited by ABGD and GMYC). In silico (relaxed) thresholds for the above ranks were also assessed by scoring taxonomic discrepancies between an OTU's best hit (obtained with function -usearch_global) and its topological reference taxonomy. From this procedure, the distribution of correctly and incorrectly classified hits was reported (for all phototrophs and the Ulvophyceae). Finally, to assess the overall performance of the database in light of annotation thresholds defined above (i.e. "How distant are OTUs from databased sequences?" and "Do abundant OTUs score highly against the database?"), the distributions of OTUs' best hit (percent identity) against the database was plotted for all phototrophic and Ulvophycean OTUs, as well as for the most abundant OTUs (i.e. those representing $\geq 1 \%$ of reads) in each of the metabarcoded samples.

\section{Availability of supporting data}

The annotated database and annotated core OTUs are available in the Dryad repository, doi:10.5061/ dryad.6cj8h. Newly barcoded specimens and clones are deposited in Genbank ${ }^{\circledR}$ (KU361834-KU362236) and the raw Illumina dataset on the National center for Biotechnology and Information (NCBI) Sequence Read Archive (\#SRP067712).

\section{Results}

\section{DNA Sequencing}

A total of 316 specimens were barcoded with tufA (including 238 Ulvophyceae, 72 Florideophyceae, and six Phaeophyceae) among which 104 were new representatives of 'Ostreobium' spp. (47 from cultures) (Additional file 1: Table S1, Additional file 2: Table S2). Cloning resulted in the sequencing of 153 bacterial colonies, which after dereplication represented a library of 86 unique tufA sequences, including 37 Chlorophyta, 25 Cyanophyta, 15 Rhodophyta, one Haptophyta and eight Ochrophyta (see Additional file 5: Table S5 for class details). The shared Miseq run output was a total of 4,917,888 raw paired-end reads for the four microfloras tested. Upon data processing throughout the USEARCH pipeline, read numbers gradually decreased, leaving $27 \%$ of the raw data for OTU abundance mapping and $\sim 17 \%$ for clustering (Table 4). Clustering at multiple levels with SWARM ( $\mathrm{d}=1-16)$ output from 2483 to 740 OTUs. SWARM indicated a clear clustering optima between local thresholds of $\mathrm{d}=10-13$ (i.e. up to 13 steps from a given centroid), where the number of common OTUs with UPARSE did not vary (Table 5). Clustering at the $97 \%$ recommended
Table 4 OTU counts and corresponding read counts per microflora sample

\begin{tabular}{lrr}
\hline & OTUs & \multicolumn{1}{c}{ Reads } \\
\hline Mapped & 802 & $1,261,195$ \\
Corrected $^{\mathrm{a}}$ & 802 & $1,260,811$ \\
FL01 & 28 & 23,785 \\
FL02 & 317 & 558,503 \\
JP07 & 609 & 672,119 \\
GM14 & 38 & 6404 \\
\hline
\end{tabular}

a Table cells with read counts $<3$ were removed

global threshold with UPARSE generated 822 OTUs, the majority but 20 were also produced by SWARM. The 802 core OTUs common to SWARM and UPARSE mapped to $1,260,811$ ( $94 \%)$ of the $1,331,438$ reads available (70,243 reads unmapped) (Table 4). The number of OTUs and their final read counts for each of the microflora samples clearly reflected the differential sequencing depth carried at MRDNA. Non-cumulative ranked abundance curves demonstrated 3-5 OTUs strongly dominating each of the microfloras and the presence of numerous low abundance OTUs (i.e. long tails, Fig. 5). Cumulative curves (ranked OTUs) demonstrated rapid plateauing of mapped read abundance (Fig. 5 and Additional file 6: Figure S1). Phototrophs represented 618 out of the 802 robust OTUs, i.e. $77 \%$ of OTUs (53.3-84.6 \% depending on the sample) and overall $98 \%$ of mapped reads (83.2-99.4\% depending on the microflora sample) (Fig. 13, Additional file 7: Table S6).

\section{Phototroph phylogeny}

Phylogenetic reconstruction of phototrophic tufA diversity recovered topological features congruent with those presented in Delwiche et al. [19], albeit with much denser taxon sampling. The resulting tree was characterized by a poorly resolved backbone with higher resolution at lower taxonomic levels. Phylum para/polyphyly was caused by the nesting of secondary endosymbiotic phyla (Ochrophyta, Haptophyta, Cryptophyta; and Euglenophyta and 'Chlorarachniophyta') among extent members of early (single-celled) lineages of the Rhodophyta and Chlorophyta [46] from which they diversified (respectively). A few classes are also para/polyphyletic in congruence with known taxonomic discrepancies (e.g. see [34]). Subclasses of the Cyanophyta (not shown) are also mostly polyphyletic (e.g. Synechococcophycideae) and distributed between two major clades (as in e.g. [63, 83]). Overall, the phototrophic diversity comprised in the database includes 27 classes in 10 phyla (Fig. 6, Additional file 4: Table S4). Several phylogenetic landmarks in the evolution of photosynthetic organisms are highlighted on the tree (Fig. 6). 
Table 5 Number of OTUs common to UPARSE and SWARM output at multiple clustering thresholds

\begin{tabular}{|c|c|c|c|c|c|c|}
\hline & UPARSE & $99 \%$ & $98 \%$ & $97 \%$ & $96 \%$ & $95 \%$ \\
\hline SWARM & & 1070 & 918 & 822 & 761 & 698 \\
\hline d01 & 2483 & 1044 & 913 & 818 & 752 & 696 \\
\hline d04 & 1416 & 963 & 902 & 813 & 750 & 693 \\
\hline d08 & 1208 & 851 & 845 & 806 & 748 & 690 \\
\hline d09 & 1135 & 835 & 829 & 804 & 747 & 689 \\
\hline d10 & 1093 & 833 & 827 & 802 & 747 & 689 \\
\hline d11 & 1066 & 833 & 827 & 802 & 747 & 689 \\
\hline$d 12$ & 1047 & 833 & 827 & 802 & 747 & 689 \\
\hline$d 13$ & 1032 & 833 & 827 & 802 & 747 & 689 \\
\hline d14 & 788 & 735 & 735 & 735 & 719 & 676 \\
\hline d15 & 763 & 722 & 722 & 723 & 708 & 673 \\
\hline d16 & 740 & 706 & 706 & 707 & 696 & 668 \\
\hline
\end{tabular}

Recommended global threshold for UPARSE (97\%) and determined local threshold optima for SWARM (d10-d13) are in bold italics. The 802 OTUs kept for subsequent analyses are italicized

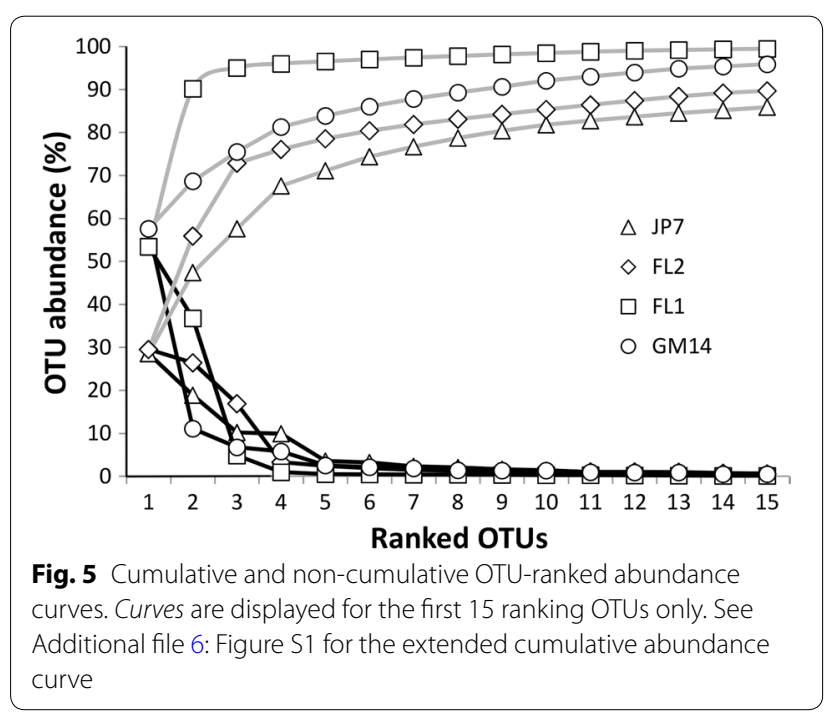

\section{OTU richness and abundance}

The phylogenetic distribution of OTUs (richness and abundance) is clearly visible on the phototroph tree (see dashed lines and bars' height, Fig. 6) and allowed their classification (on a topological basis) to 14 classes in seven phyla. All but 22 eukaryotic OTUs could not be assigned with confidence to a specific class within the phylum they branched in (Additional file 8: Table S7); however, these OTUs represented very few reads $(<0.25 \%$ of all mapped reads). The densely colonized microfloras FL02 and JP07 (i.e. those sequenced deeply) exhibited particularly similar richness profiles for phylum, class, and Ulvophycean family (Fig. 13). Together, these two microfloras harbored 605 phototrophic OTUs, 141 of which were assigned to both samples following demultiplexing (Additional file 9: Figure S2, Additional file 8: Table S7). Their abundance profiles were also similar except at the family level (Fig. 13). By comparison, the less dense microfloras FL01 and GM14 (i.e. those with low sequencing depth) showed skewed profiles toward particular groups and much fewer OTUs (33 phototrophic OTUs, 13 unique to these two samples, 20 shared with others). In the coral reef samples (i.e. FL01, FL02, and JP07), the Cyanophyceae (Cyanophyta) and 'Bacillariophyta' (Ochrophyta) were found in low read abundance but revealed tremendous OTU diversity (267 and 162 OTUs, respectively), while the Coccolithophyceae (Haptophyta), the Pedinophyceae and Ulvophyceae (Chlorophyta) and the Florideophyceae (Rhodophyta) were found in large read abundance but were much less diverse (i.e. few OTUs, Fig. 6). In the rhodolith sample (GM14), the Florideophyceae were particularly abundant.

\section{Ulvophyceae phylogeny}

The phylogenetic estimation made on the Ulvophyceae dataset (Fig. 7) resulted in nearly identical branching features than within the larger tree except for a few differences in the Bryopsidales (shown for the Ostreobidineae, see Fig. 8). The Bryopsidales and the 'UlvalesUlothrichales' radiations each received strong bootstrap support (95 and $82 \%$, respectively). Overall, the tree details 23 families, some accepted taxonomically, others provisionally delimited here based on topological features and clade divergence. 'Ostreobium' is revealed as a polyphyletic form genus representing a complex of family-level clades (Figs. 7, 8, 9). Most of its diversity is monophyletic within a large, confirmed subordinal-level clade, the Ostreobidineae (previously proposed by [102]), whose divergence is particularly consistent with other 


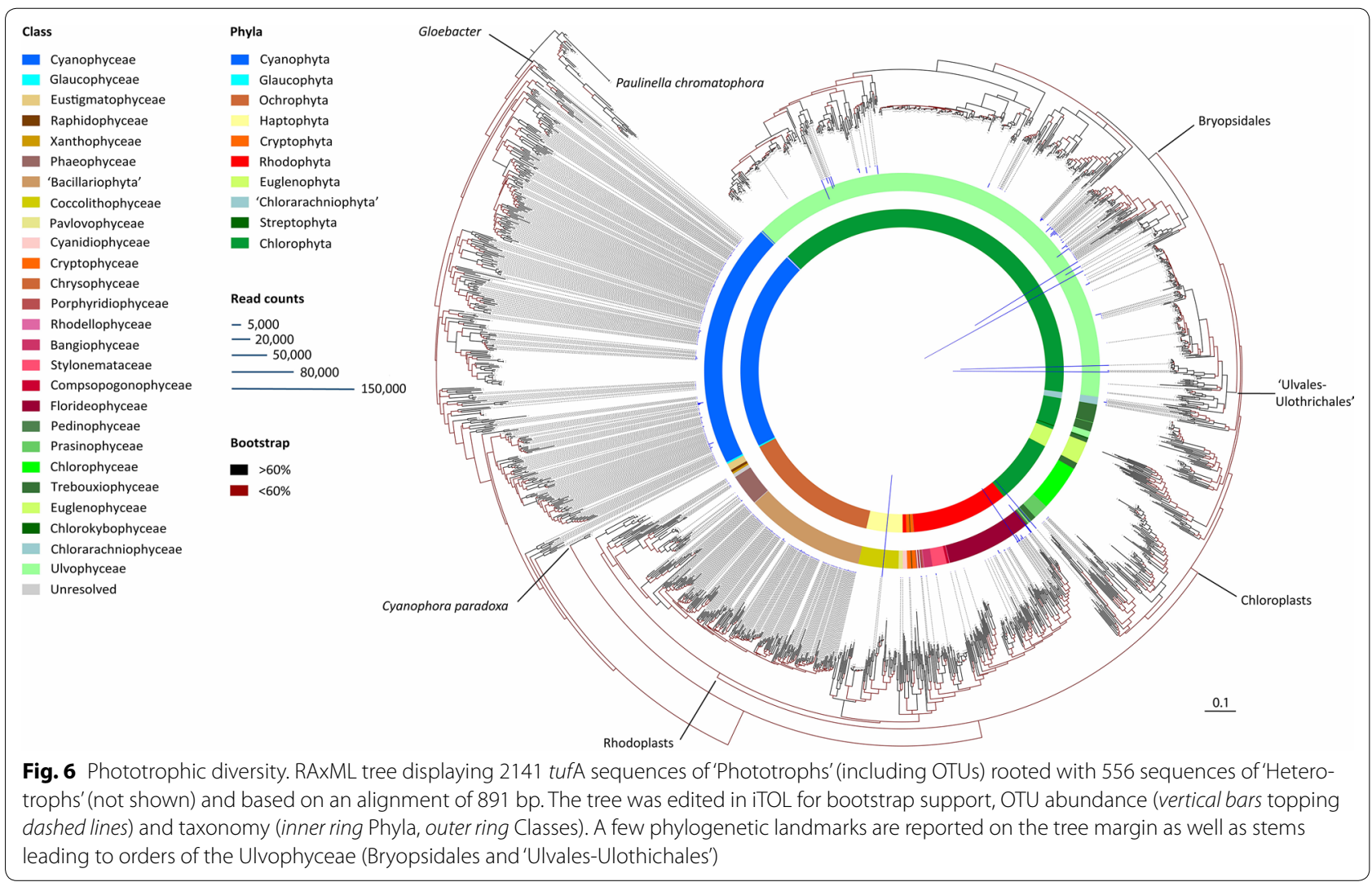

suborders, the Bryopsidineae and the Halimedineae (Fig. 9). In this suborder, four 'Ostreobium' families are provisionally delimited and named from their location of collection in the Ryukyus for the purpose of molecular classification (nomina nuda 'Hamidaceae', 'Maedaceae', 'Odoaceae' and 'Unarizakiaceae', Fig. 9). Few OTUs are found in the phylogenetic vicinity of the 'Maedaceae' and presently cannot be assigned to a family clade (Figs. 7, 8). Additional 'Ostreobium' diversity is found in a remote family-level clade of the suborder Halimedineae provisionally assigned for taxonomic simplicity to the 'Pseudostreobiaceae' (Figs. 7, 8, 9). Our barcoding efforts also further documented members of the diminutive epilithic, polyphyletic form genus 'Pseudochlorodesmis' delimited here in two provisionally family-level clades named based on their taxonomic history [103], i.e. the 'Pseudochlorodesmidaceae' and Siphonogramenaceae' (Figs. 7, 8, 9). Other 'Pseudochlorodesmis' spp. (see Additional file 1: Table S1 for details) represented primordia of otherwise conspicuous taxa in families of the Halimedineae (e.g. in Halimeda, see [64]) and neotenic thalli within the Rhipiliaceae [103] and the Caulerpaceae (a sister taxon to the C. 'ambigua' complex, [23]). Barcoding of diminutive, simple Bryopsidineae also revealed numerous unresolved/unknown taxa in the phylogenetic vicinity of the
Derbesiaceae and Bryopsidaceae (Figs. 7, 8). Several epilithic families are found endolithically as seen by the presence of OTUs in their clade (Fig. 7, e.g. the Bryopsidaceae, Derbesiaceae and Halimedaceae) supposedly as reproductive cells, germlings and/or endolithic siphonous networks).

\section{Delimited species}

The GMYC analysis delimited 504 hypothetical species in the Ulvophyceae (LGMYC $=7663.072>\mathrm{LO}=7$ 577.771.182, $\mathrm{P}=0$ ) while with ABGD the output was 666 spp. Species groups delimited by the two methods (GMYC-ABGD) represented 349-485 spp. in the Bryopsidales, and 147-172 spp. in the 'Ulvales-Ulothrichales' (Fig. 7). The majority of discrepancies arose in the Bryopsidales in well-sampled families of the suborder Halimedineae (overall 195-308 spp.), namely in the Halimedaceae (56-88 spp.) and especially in the rapidly diversifying Caulerpaceae (67-132 spp.), where GMYC seemed to overlump and ABGD oversplit terminal clades. By contrast, species delimitation showed greater congruence between the two methods in the remainder of the Ulvophyceae, in the 'Ulvales-Ulothrichales' (Ulvellaceae: 56-65 spp., Ulvaceae: 43-52 spp., Phaeophilaceae: 13-18 spp.) and in the Bryopsidales within the suborders 

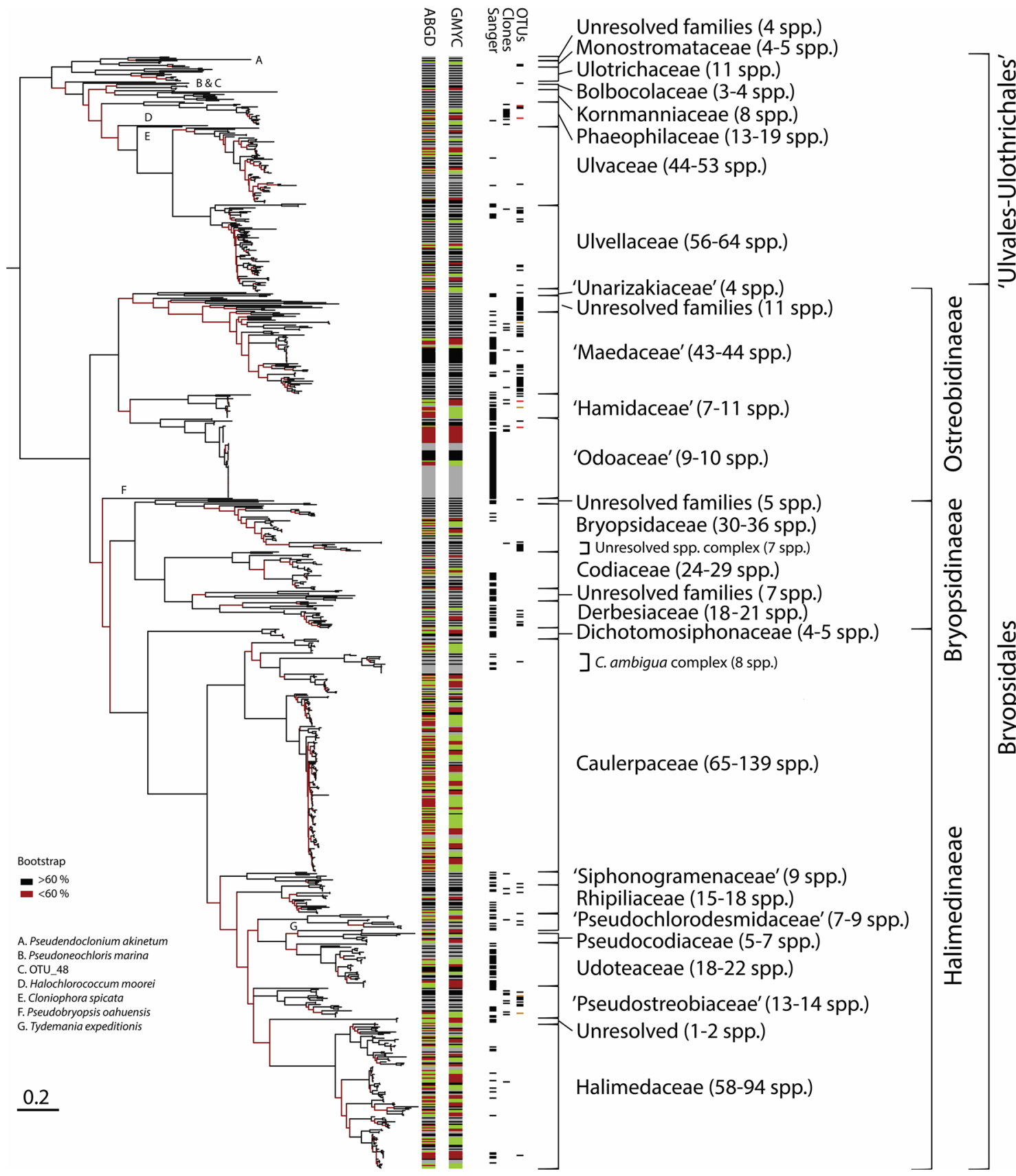

Fig. 7 Ulvophyceae diversity. RAxML tree displaying 901 tufA sequences of the order Bryopsidales and 'Ulvales-Ulothrichales' based on an alignment of $891 \mathrm{bp}$. The tree was rooted with few outgroup taxa of the Chlorophyta and edited in iTOL for bootstrap support, molecular species, and sequencing method (Sanger, Cloning or Metabarcoding). OTU abundance color-coded for high read counts (red), moderate (brown) and low (black). Congruent clusters of molecular species between ABGD and GMYC are represented in alternating dark and light grey colors. Incongruent species boundaries are displayed in alternating colors yellow and red. Taxonomy is reported on the tree margin. Note important incongruence in molecular species delimitation between the two methods in the families Caulerpaceae and Halimedaceae

Bryopsidineae (overall 85-91 spp.) and Ostreobidineae (overall 72-81 spp.; 53-58 spp. in the Ryukyus alone). The remote Ostreobium clade found in the Halimedineae, i.e. the 'Pseudostreobiaceae', harbors 13-14 spp.
Molecular species divergence computed over the metabarcode length (375 bp) indicated species boundaries lying between 99.73 and $97.87 \%$ with the GMYC method (i.e. 1-8 bp substitutions), and between 99.73 and $99.47 \%$ 


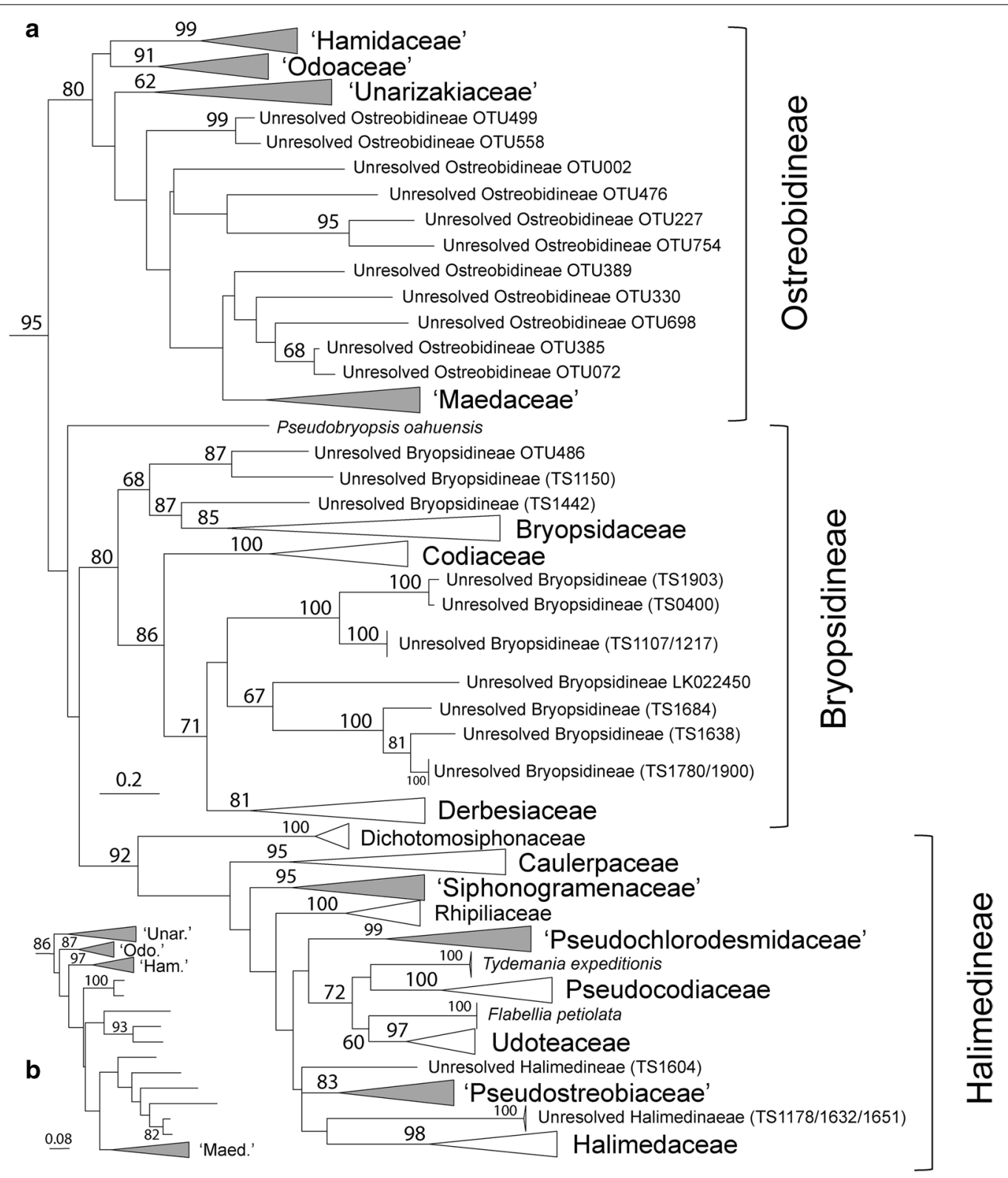

Fig. 8 Family diversity in the order Bryopsidales. a Summary family topology of the Bryopsidales obtained with RAxML for the Ulvophyceae data set (see Fig. 7). b Close-up of alternative family branching order in the Ostreobidineae obtained with RAxML for the full database tree (magnified from Fig. 6). Families provisionally delimited in the present study are shaded in gray

with ABGD (1-2 bp substitutions) (not shown, see Additional file 10: Figure S3).

\section{Identity Threshold for annotation}

Examination of taxonomic rank divergence obtained with sppDist within the Ulvophyceae (Fig. 10) indicated conservative annotation thresholds for family, suborder and order boundaries at 92,84 and $79 \%$, respectively (values rounded up from 91.2, 83.2 and $78.9 \%$, respectively) and relaxed annotation thresholds at $85,79,77 \%$, respectively (values rounded up from 84.5, 78.4, and $76.3 \%$ ) (Fig. 11). For the full breadth of phototrophic OTUs, conservative annotation thresholds for class, phylum and domain boundaries laid at 86,83 and $82 \%$, respectively (values rounded up from 85.1, 82.2 and $81.9 \%$ ), while relaxed annotation thresholds laid at 84,83 and $77 \%$, respectively (values rounded up from 84.0, 82.7 and $76.3 \%$ ) (Figs. 10, 11, respectively). Overall, phototrophic OTUs matched 


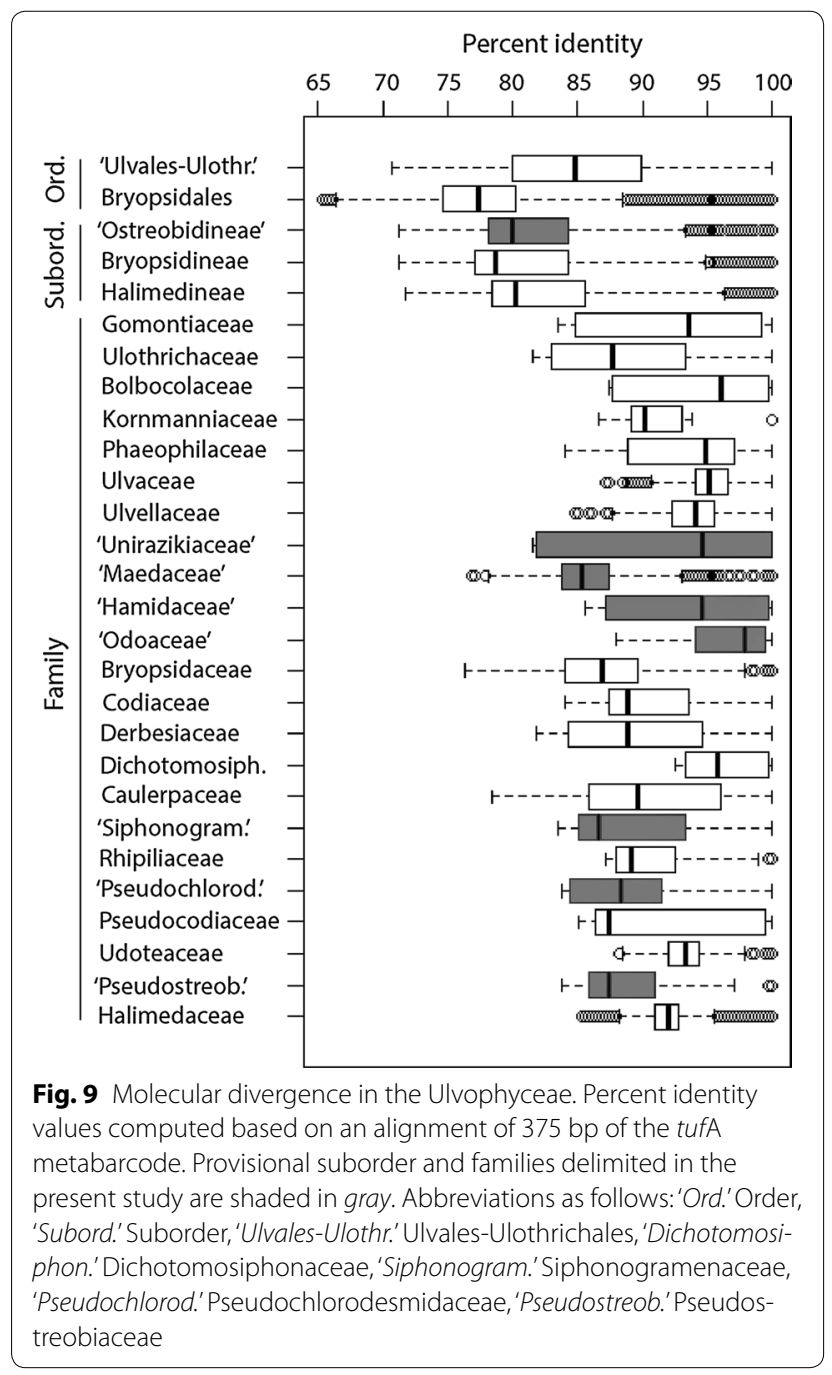

the database at high identity levels with the majority of hits $\geq 84 \%$ (see lower boxplot quartiles, Fig. 12) in the range of class annotation (i.e. relaxed $=84 \%$, conservative $=86 \%)$. For the Ulvophyceae, the majority of hits were $\geq 87 \%$ (see lower boxplot quartiles, Fig. 12) in the range of family-level thresholds (i.e. relaxed $=85 \%$, conservative $=92 \%$ ). The most abundant OTUs, those driving abundance profiles (i.e. $>1 \%$ abundance, Fig. 13 and Additional file 11: Figure S4), hit the database at even higher identity values (lower quartile $>91 \%$, Fig. 12) and displayed the same distribution for the phototrophs ( $\mathrm{n}=32$ OTUs) or the Ulvophyceae-only ( $\mathrm{n}=19$ OTUs).

\section{Discussion}

\section{Bioinformatics}

Following demultiplexing, we noted important read losses ( $>50 \%$ ) (Table 3 ) probably caused by the systematic exclusion of reads containing base pair error(s) within their index in the early steps of the UPARSE pipeline

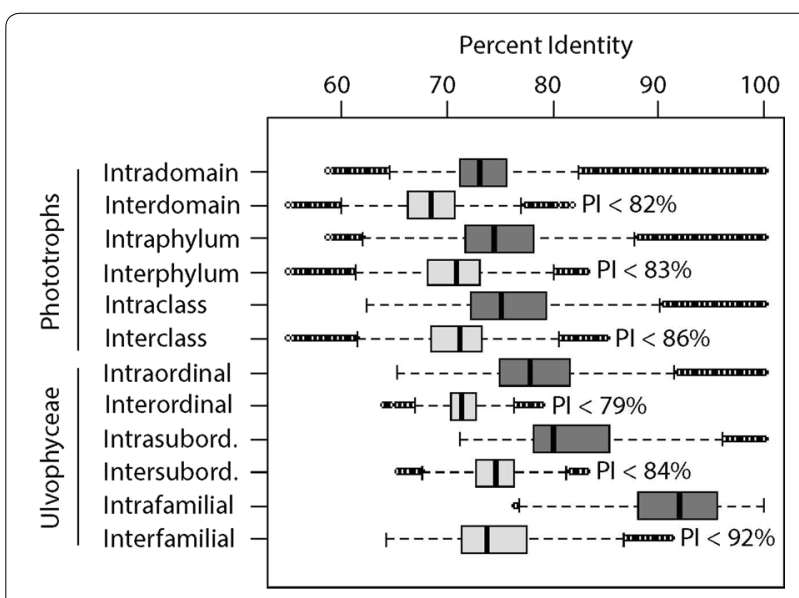

Fig. 10 Conservative annotation thresholds. Overall intra- and intermolecular divergence (dark and light grey, respectively) at the domain, phylum and class levels for all phototrophs and at the ordinal, subordinal and family levels within the Ulvophyceae (orders Bryopsidales and 'Ulvales-Ulothrichales'). Percent identity values were computed based on an alignment of $375 \mathrm{bp}$ of the tufA metabarcode. Minimum conservative thresholds for metabarcode classification/annotation are printed. $P$ I percent identity

(no mismatch allowed). Here, demultiplexing losses may have also been exarcerbated by the length of our amplicon $(467 \mathrm{bp})$ for the chemistry used $(2 \times 250 \mathrm{bp})$ since paired-ends could not overlap over the index regions. An additional consequence of potential increased substitution errors in the index region along with tag jumping in Illumina-based metabarcoding [89], is the misassignment of sequence-to-sample (i.e. the false assignment of an OTU to a given sample), which may inflate OTU diversity across samples and thus bias richness profiles. However, since index error/jumping generally concerns only a small proportion of reads, false assignment is less likely to bias abundance profiles (Fig. 13), especially when those profiles are underlayed by very abundant OTUs such as shown here (Fig. 5 and Additional file 11: Figure S4). Although we noted some possible tag error/ jumping in OTUs retrieved in the deeply sequenced microfloras FL02 and JP07 (Additional file 12: Figure S5), numerous unique OTUs were also found in each of these samples (Additional file 9: Figure S2), (Fig. 13). If there is any bias, we suspect that the richness profile reported for the Ulvophyceae (Fig. 13, bottom left histogram) was the most affected since this class included OTUs found in very large proportion of reads that were more likely to propagate ('bleed') to other samples via tag error/jump. Hence, in this regard, abundance profiles are in our opinion a much more robust depiction of OTUs actually present in a given sample.

Currently, tag-jumps represent a non-negligible issue of the metabarcoding approach. Indeed, Esling et al. [28] 


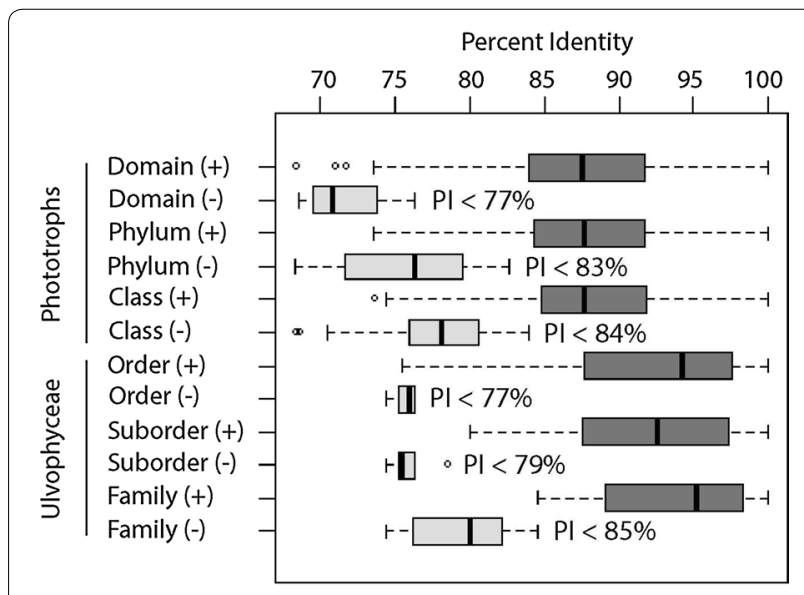

Fig. 11 Relaxed annotation thresholds. Distribution of taxonomic match $(+) /$ mismatch $(-)$ between metabarcodes' best hit and their tree-based classification for all phototrophs and the Ulvophyceae. Percent identity values were computed based on 375 bp of the tufA metabarcode. Minimum thresholds to avoid misclassification are printed. $P$ I percent identity

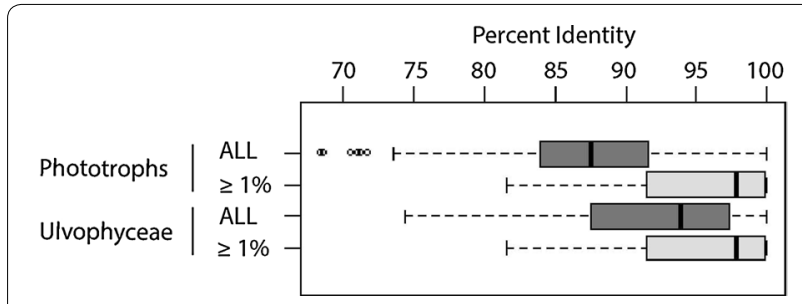

Fig. 12 Database performance. Distribution of OTU's best hit against the database for phototrophs and for the Ulvophyceae-only (orders Bryopsidales and 'Ulvales-Ulothrichales'). 'ALL' represents matches for all OTUs regardless of abundance (in dark grey). ' $\geq 1$ \%' represents matches for the most abundant OTUs (in light grey). Note the skewed distribution toward high identity matches for the most abundant OTUs (regardless of taxonomy) indicating high performance of the database for annotation of the targeted communities. Values were computed based on 375 bp of the tufA metabarcode

demonstrated that libraries prepared with single index primers (as done here) and saturated double index primers result in undetectable read cross-contamination. This is particularly problematic when presence-absence of OTUs (i.e. richness) is critical for the outcome of a particular study (e.g. keystone OTU, geographic variation). To mitigate cross-contamination, these authors showed that multiplexing samples following Latin Square designs can optimize mistag detection. Recently, Kitson et al. [48] also reported on the development of nested metabarcode tagging $(2 \times 2$ indexes) that apparently lead to accurate sample/index tracking. Sequencing depth and evenness of the community found within a sample are also nontrivial issues affecting community profiles (richness and abundance) obtained via metabarcoding. On the one

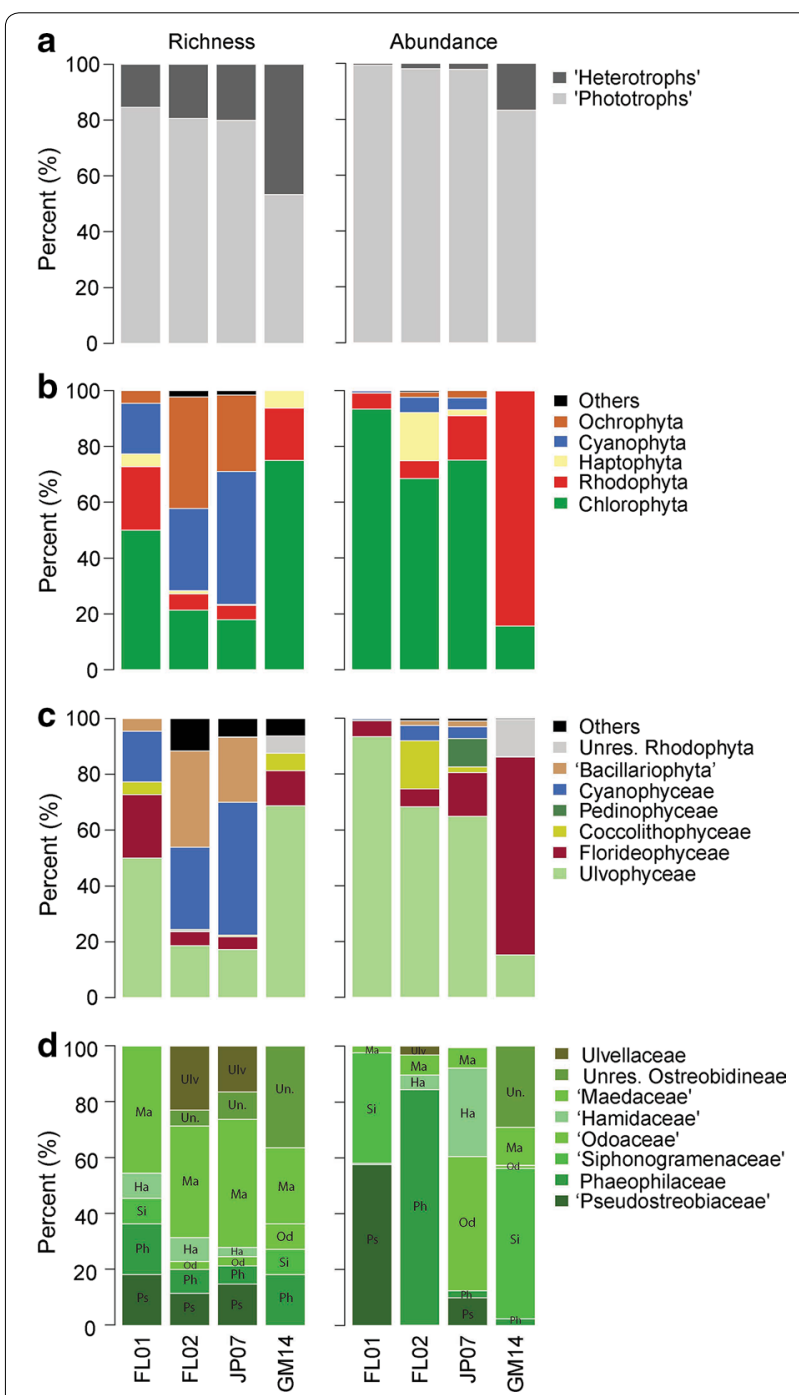

Fig. 13 OTU richness and abundance at multiple taxonomic levels. a 'Phototrophs' vs. 'Heterotrophs', b photrophic phyla, c phototrophic classes, and $\mathbf{d}$ Ulvophycean families within orders Bryopsidales and 'Ulvales-Ulothichales'. Richness represents OTU diversity as percentage of the total number of OTUs. Abundance represents OTUs read counts as percentage of total mapped reads per sample. Family abbreviations as follows: 'Ulv' Ulvellaceae, 'Un.' Unresolved Ostreobidineae, 'Ma' Maedaceae, 'Ha' Hamidaceae, 'Od' Odoaceae, 'Si' Siphonogramenaceae, 'Ph'Phaeophilaceae, 'Ps' Pseudostreobiaceae. 'Others' includes very low abundance representatives (each $<1 \%$ of mapped reads in average) of phylum Cryptophyta and 'Chlorarachniophyta' and the classes Bangiophyceae, Compsopogonophyceae, Chlorarachniophyceae, Cryptophyceae, Pavlovophyceae, Phaeophyceae, Prasinophyceae, Stylonematophyceae and unresolved members of the Chlorophyta, Haptophyta, and Ochrophyta (See Additional file 7: Table S6, Additional file 8: Table S7; Additional file 11: Figure S4 for further details)

hand, samples sequenced evenly (i.e. normalized across samples for similar sequencing depth) may not show all diversity present for samples that are strongly dominated by a few taxa 'masking' low abundance OTUs [2]. On the 
other hand, samples sequenced unevenly (as done here) may falsely exhibit higher diversity simply because they were sequenced at greater depth, which promotes biodiversity recovery [91]. In spite of these concerns, we are confident that our deeply sequenced samples JP07 and FL02 truly harbor more diversity than FL01 and GM14, especially considering the dense endolithic colonization observed for these samples, and that the preparation of JP07 consisted in pooling subsamples of microfloras from multiple locations across the Ryukyus archipelago to specifically maximize biodiversity recovery (see "Methods" section). To further support the above, we subsampled JP07 and FL02 to a shallow sequencing depth of 20,000 reads as reported for FL01 and GM14 (Table 4). Following OTU clustering (not shown), we observed a $\sim 2-3$ fold higher diversity for JP07 and FL02 than for FL01 and GM14, namely 87 and 66 OTUs vs. 28 and 38 OTUs, respectively. We nonetheless recognize that sequencing FL01 and GM14 deeper could have retrieved some additional OTU diversity.

Overall, sequencing future projects with the now available $2 \times 300$ bp chemistries should greatly improve upon demultiplexing issues caused by index errors. In spite of the noted important read losses, our data set contained very high read redundancy and still comprised important sequencing noise (as seen from overly abundant singletons, doubletons and tripletons resulting from the deep sequencing of FL02 and JP07, Table 3) and thus included much sufficient data to assess our microfloras' diversity. Using a combination of superior algorithms with different de novo clustering strategies (as recommended in [88]) such as UPARSE's global and SWARM's local threshold algorithms, allowed us to filter for high quality core OTUs produced by both pipelines. As a result of this dual-clustering approach, our OTUs are very close to biological sequences (i.e. contain 0,1 or 2 incorrect bases as shown for clones and Illumina core OTUs obtained for JP07, our only sample sequenced with both methods, (see Additional file 13: Figure S6), in congruence with the $\leq 1 \%$ error rate reported on a mock community for UPARSE alone [26]. Overall, the phylogenetic breadth documented by our de novo OTUs (prokaryotic and eukaryotic diversity), added to the negligible proportion of identified contaminants (i.e. non-tufA, $<1 \%$ of filtered reads, Table 3), together demonstrate the specificity of our newly designed degenerate primers to target tufA from phototrophs that represented the majority of retrieved OTUs (77\% overall) and mapped reads (>98\% overall) (Fig. 13 and Additional file 7: Table S6). The priming region of env_tufAF (Fig. 4) actually overlaps with an extended stretch of codon insertion/deletions in numerous heterotrophs, which may further favor annealing, and therefore amplification, toward phototrophic diversity. For samples with very low phototroph content and/or containing inhibitors (i.e. secondary metabolites [105]), target capture via hybridization with tufA probes may provide a valuable alternative to amplification, as well as enhance rare OTUs detection limits. Some studies have performed target capture metabarcoding, such as for instance Patel et al. [71], Denonfoux et al. [20] and more recently Dowle et al. [22].

\section{Endolithic Ulvophyceae}

The molecular diversity and phylogenetic breadth of the boring microsiphons referred to as Ostreobium spp. had clearly been overlooked in the literature and lacked tufA referencing (a single sequence-FJ535859 was available on GenBank ${ }^{\circledR}$ prior to our study). We document for this form genus an impressive polyphyletic cryptic diversity encompassing an estimated 85-95 species-level entities (Fig. 7), for which we delimited five provisional families to facilitate the profiling of endolithic communities (Figs. 9, 13). These families exhibit molecular divergence comparable to conspicuous Bryopsidalean families that are well circumscribed morphologically (e.g. the Halimedaceae, the Caulerpaceae, etc., Fig. 9). Our phylogeny also reveals unresolved branches at the base of the 'Maedaceae' (Ostreobidineae) (Figs. 7, 8), which pending further sampling, may lead to the delimitation of additional families. In this endeavor, clarifying the molecular identity of the generitype O. quecketti [7] from its type locality (Le Croisic, Brittany, France) should allow recircumscription of the family Ostreobiaceae P.C. Silva (proposed in [70]; validated in [9]) toward establishing a more stable classification. We expect that sequencing of endolithic communities worldwide will further increase species diversity of euendolithic microsiphons. Finally, although field collections were focused on 'Ostreobium' spp., our assessment also recovered common euendolithic microfilamentous genera of the 'Ulvales-Ulothrichales', namely the Phaeophilaceae and Ulvellaceae, in culture and from cloning and metabarcoding (Fig. 7, Additional file 1: Table S1, Additional file 5: Table S5 and Additional file 8: Table S7).

\section{Database performance}

Thorough analyses of our newly established database resources for tufA permitted referencing the taxonomy of OTUs based on their phylogenetic position, as well as defining identity thresholds for their rapid annotation in the context of environmental biomonitoring studies via metabarcoding. Although the backbone of our phototroph tree was overall poorly resolved (Fig. 6, as in [19]), topological relationships obtained among phyla/classes with tufA were congruent with currently accepted hypotheses of chloroplasts/rhodoplasts and secondary endosymbiotic lineages evolution [74], allowing visualization of the 
database and OTUs in a phylogenetically sound framework (Fig. 6 and Additional file 14: Figure S7). Relying on our divergence analyses, we were able to establish in silico (relaxed) and clade-based (conservative) thresholds for OTU annotation at high taxonomy levels (Class $84-86 \%$, Phylum 83-83 \%, and Domain 77-82 \%, Figs. 10, 11) and lower taxonomy levels in the Ulvophyceae (species 97-99 \%, family $85-92 \%$, suborder 79-84 \% (for the Bryopsidales) and order $77-79 \%$, Figs. 10, 11). The fact that high-level taxonomy thresholds exceeded lower-level ones (i.e. within the Ulvophyceae) reflects the combination of group-specific differences in tufA's evolutionary rate (e.g. due to genome architecture, or lineage diversification age), low biodiversity sampling in some clades, taxonomic discrepancies, and poorly resolved relationships (i.e. paraphyletic/polyphyletic classes and phyla). Overall, our database achieved matching of OTUs at high identity values (the majority matching at $>85 \%$, Fig. 12), which as a single threshold holds the potential to accurately annotate OTUs to both family (for Ulvophyceae representatives) and class-levels (all phototrophs). Here, the majority of hits below $85 \%$ belonged to the class Cyanophyceae (not shown), which as the sole representative in the phylum Cyanophyta (and 'Phototrophs' in the prokaryotic domain) may thus be annotated with identity thresholds of $82 \%$ (Fig. 10), and eventually further relaxed down to $77 \%$ (Fig. 11). Overall, we recommend applying a range of thresholds (based on the above relaxed and conservative thresholds) and investigate with tree methods the phylogenetic position of OTUs that may cause profile disparities in order to correct their taxonomy. As demonstrated in the present study, our 375 bp metabarcode packs sufficient phylogenetic signal for model-based tree estimation when included in well-sampled sequence alignments (e.g. as implemented here within RAxML). Finally, matches in the 97-99 \% range could be used for molecular species annotation in the Ulvophyceae (as estimated by GMYC or ABGD analyses, see Fig. 7 and Additional file 10: Figure S3), providing that profiling at this level becomes necessary and that a molecular-based taxonomy becomes available for this cryptic diversity (e.g. [44]).

\section{Community profile}

In spite of a possible, minimal bias in richness profile introduced via tag error/jump, the profiling of the microfloras revealed a core assemblage of phyla, classes and some Ulvophycean families inhabiting endolithic communities, whose abundance varies, but appears universal (Fig. 13). Similarities in the phyla/classes richness and abundance profiles of the OTU-rich coral reef samples FL02 and JP07 are interesting considering their distant geographic origin (Pacific vs. Atlantic) and the fact that for the preparation of JP07, multiple $\mathrm{CaCO}_{3}$ samples collected from across the Ryukyu archipelago had been pooled together to maximize the recovery of endolithic biodiversity (see "Methods" section). In this regard, JP07 assemblage may thus be viewed as an average phyla/classes profile for shallow coral reefs of the Ryukyus (e.g. abundance of $<5 \%$ 'Bacillariophyta', $<5 \%$ Coccolithophyceae, $<5 \%$ Cyanophyceae, $<20 \%$ Florideophyceae and $>65 \%$ Ulvophyceae, and miscellaneous groups), whose applicability to other tropical areas such as in the Atlantic (FL02) would appear relevant. By contrast with these two samples, the skewed profiles of the less densely colonized microfloras FL01 and GM14 may reflect immature or developing communities undergoing succession following for example, recent niche opening or disturbance. The rhodolith microflora of GM14 was particularly Rhodophyta-rich from an abundant crust-forming Rhizophyllis sp. OTU (Florideophyceae) and an early branching OTU that we could not presently resolve within the phylum (Fig. 13, Additional file 11: Figure S4). Although we were careful to sample $\mathrm{CaCO}_{3}$ patches devoid of conspicuous encrusting red algae for the preparation of the microfloras' DNA extracts, we cannot exclude that accidental drilling of Rhizophyllis (Rhizophyllidaceae) (present on the surface of GM14 upon this sample reexamination) could account for these reads rather than propagules of this taxon present endolithically. Likewise, the most abundant Rhodophyta in other microflora samples were also encrusting taxa, the families 'Corallinaceae-Hapalidiaceae' and Peyssonneliaceae (Florideophyceae), but other non-encrusting taxa were also present (in class Bangiophyceae, Compsopogonophyceae, Stylonematophyceae, and within the Florideophyceae, e.g. the order Ceramiales, and family Dumontiaceae, Additional file 7: Table S6, Additional file 8: Table S7). Lastly, GM14 also exhibited a lower phototroph/heterotroph ratio than other microfloras (Fig. 13), which may be explained by its depth of collection in the mesophotic zone $(65 \mathrm{~m})$ where light availability may limit photosynthesis and algal $\mathrm{CaCO}_{3}$ penetration more importantly than in shallow waters.

\section{Evolutionary perspectives}

Our barcoding approach coupled with metabarcoding brought to light the widespread use of the endolithic niche as a habitat by the Bryopsidales (including otherwise epilithic taxa, see OTU and clones' distribution in Fig. 7). For instance, several macroscopic taxa of the Bryopsidineae (e.g. Derbesiaceae, Bryopsidaceae) and the Halimedineae (e.g. Caulerpaceae, Halimedaceae, Rhipiliaceae, 'Siphonogramenaceae and Pseudochlorodesmidaceae') are present and may occupy the substratum in the form of germlings, reproductive cells, and endolithic siphonous/microsiphonous networks, the latter possibly used 
for lateral vegetative dispersal, thallus regeneration and access to nutrient-rich sediment trapped in cavities. Overall, the Bryopsidales seem to present a tight evolutionary link with the endolithic niche as an ancestral habitat, as evidenced by the early branching of the Ostreobidineae in multi-marker studies [102], also supported here with tufA (bootstrap $>95 \%$, Fig. 8). We hypothesize that environmental constraints associated with the epilithic niche such as high light/UV, direct water flow, and macroscopic predators (which themselves diversified and specialized over time, [16], such as kleptoplastic sacoglossan sea slugs, [39]) may have sparked the diversification of morphologically complex and conspicuous families from ancestral microsiphons living at the interface between the water column and the endolithic niche. In epilithic families able to exploit the endolithic niche with networks of siphons, this feature may thus represent a symplesiomorphy for some (e.g. the 'Siphonogramenaceae and 'Pseudochlorodesmidaceae') and a possibly re-acquired trait for others (i.e. the Caulerpa 'ambigua' complex in the Caulerpaceae) as suggested from the long branches leading to such lineage (i.e. accelerated evolution following key innovation into a new adaptive zone, Simpson [92, 93]) (Figs. 7, 8). Aside from the Bryopsidales, the phylogenetic extent of phototrophic OTUs also found in the endolithic niche seemingly in the form of chaesmo- and cryptoendolithic reproductive cells or germlings, perennial boring euendoliths (endolithic Ulvophyceae) as well as transient ones stages (e.g. some alternate life history stages of the Florideophyceae, Rhodophyta), highlights the critical importance of the $\mathrm{CaCO}_{3}$ substratum in algal evolution as a seedbank for life cycle completion and survival of diverse algal taxa [30, 32].

\section{Conclusions}

In summary, we provide a flexible and comprehensive metabarcoding framework including primers, reference data and annotation thresholds for the facilitated recovery and rapid profiling of phototrophs found in endolithic communities. As a new resource for environmental biomonitoring, the framework is timely to enable the use of high throughput sequencing to accelerate biodiversity characterization of microbial/algal assemblages from endolithic communities found in coral reef and rhodolith ecosystems in the context of global change studies (e.g. bioerosion, distributional shifts), holobiont functioning and anthropogenic degradation (e.g. eutrophication, overfishing). Our framework could also find useful applications for water quality studies related to public health, such as the monitoring of river eutrophication based on the Diatom Index (i.e. composition of the 'Bacillariophyta' $[47,104])$ and the detection of toxic Cyanobacteria (i.e. the Cyanophyceae) in freshwater and coastal systems $[4,6]$ pending further curation and increase in reference data for these particular groups, which our primers retrieved very efficiently. Overall, tufA metabarcoding assays could be performed on numerous types of samples harboring algal phototrophs including for instance water column, biofilms, periphyton, soil and ice samples and herbivorous organisms (e.g. vertebrate/invertebrate stomach contents, kleptoplastidic slugs tissue). Further studies of endolithic communities in coral reef and rhodolith ecosystems may reveal potential bioindicators of ecosystem degradation (taxonomic group/OTU ratio), whose efficient monitoring and detection may allow better management and conservation practices.

\section{Additional files}

\begin{abstract}
Additional file 1: Table S1. Barcoded Ulvophyceae. Collection information for Ulvophyceae specimens newly sequenced for tufA. To ease public database searches, Genbank ${ }^{\circledR}$ identifiers include both morphological and molecular identification (in brackets [) at the genus or family level (when abbreviated). Polyphyletic genera, species complexes or combined orders indicated in between quotes. All specimens from shallow waters $(<0-5 \mathrm{~m})$ otherwise indicated in footnotes. Endolithic habitat abbreviated as follows: $\mathrm{CaCO}_{3}$ open reef substratum, Corall. Florideophycean crusts of the order Corallinales, Peyss. Florideophycean crusts of the order Peyssonneliales, LPS large polyp coral species (e.g. Galaxea sp., Favia sp.), Shell Oyster shell (primarily) or other bivalve, SPS small polyp coral species (e.g. Porites sp., Montipora sp.), na epilithic, epiphytic, or not recorded. Collector (Coll.) initials as follows: $D P=D$. Pence, $H S=H$. Spalding, JR $=J$. Richards, $\mathrm{KI}=\mathrm{K}$. Ikemoto, MDR = M. Diaz-Ruiz, MS = M. Star, NP = N. Pyron, TS $=\mathrm{T}$. Sauvage, WES $=$ W.E. Schmidt, ZM = Z. McCorkhill. Other abbreviations: Cult. = Cultured, IUI = Interuniversity Institute for Marine Science, INVEMAR = Instituto de investigaciones marinas y costeras.
\end{abstract}

Additional file 2: Table S2. Barcoded Florideophyceae, Phaeophyceae and miscellaneous taxa. Collection information for specimens of the Florideophyceae and Phaeophyceae sequenced for tufA in the present study (as well as a miscellaneous Prasinophyceae). Polyphyletic orders or family and combined orders are indicated in between quotes. All specimens from shallow waters $(0-10 \mathrm{~m})$ otherwise indicated in footnotes. Collector (Coll.) initials as follows: $C P=C$. Pueschel, $C S=C$. Stoude, $\mathrm{DG}=\mathrm{D}$. Gabriel, DK = D. Krayesky, DWF = D.W. Freshwater, $\mathrm{EC}=\mathrm{E}$. Coppejans, ED $=\mathrm{E}$. Deslandes, JC $=\mathrm{J}$. Cabioch, $\mathrm{JH}=\mathrm{J}$. Hughey, $\mathrm{JR}=\mathrm{J}$. Richards, JRu = J. Rueness, JZ = J. Zertuche, $M G=M$. Guiry, $M H H=M . H$. Hommersand, MJW = M. J. Wynne, MY = M. Yoshizaki, OC=O. Camacho, $\mathrm{SF}=\mathrm{S}$. Fredericq.

Additional file 3: Table S3. Indexed forward primers used for metabarcoding. Indexes in bold.

Additional file 4: Table S4. Summary taxonomy and content of the non-redundant tufA database. Diversity and sequence counts for (A) Heterotrophs and Phototrophs, (B) Phyla and Classes for all phototrophs and (C) Orders/suborders and families for the Ulvophyceae (Genus taxonomy not shown for conciseness). Taxonomic groupings with unsettled naming such as combined orders, provisional families, and polyphyletic families (i.e. 'Kornamanniaceae' and 'Ulothrichaceae') are noted between quotes.

Additional file 5: Table S5. Clone taxonomy. Detailed reference taxonomy for 86 phototrophic clones from microfloras sampled in the Ryukyu archipelago (JP01, JP03, JP04, JP06, JP07, JP25), an aquarium window scrap (E09) and a Gulf of Mexico crustose coralline Lithophyllum sp. rhodolith. Taxonomic groupings with unsettled naming such as combined orders, provisional families, and polyphyletic or monophyletic species or genera complexes, are noted between quotes. 
Additional file 6: Figure S1. Cumulative OTU-ranked abundance curves. Note the much larger OTU diversity in densely colonized JP07 and FL02 vs. lightly colonized microfloras FL01 and GM14. Cumulative abundances values below $80 \%$ not displayed for figure succinctness.

Additional file 7: Table S6. Summary of microfloras richness and abundance at multiple taxonomic levels. (A-B) OTU counts and read abundance for 'Phototrophs'vs. 'Heterotrophs' group. (C-D) OTU counts and read abundance for phototrophic phyla and classes. (E-F) OTU counts and read abundance for Ulvophyceaen families. Note that OTUs may be found more than once across samples.

Additional file 8: Table S7. OTUs taxonomy and their microflora assignment. Detailed reference taxonomy for 802 core OTUs as determined from their phylogenetic position (see Fig. 6). Taxonomic groupings with unsettled naming such as combined orders, provisional families, and polyphyletic or monophyletic species or genera complexes, are noted between quotes.

Additional file 9: Figure S2. Overlapping OTU diversity. Venn diagram depicting common OTU diversity (i.e. at the circles' intersection) and unique OTUs in each of FL02 and JP07 for all phototrophs and several OTU-rich classes. Overlapping OTUs may represent diversity that is both truly found in both samples (i.e. pantropical) and resulting from false assignment caused by index tag jumping or tag error (see Additional file 12: Figure S5), which may potentially inflate richness estimates. Note, however, the still important OTU richness unique to both FL02 and JP07, especially the OTU-rich classes Cyanophyceae and 'Bacillariophyta'.

Additional file 10: Figure S3. Molecular species divergence in the Ulvophyceae. Distribution of intra- and interspecific divergence for molecular species of the orders Bryopsidales and 'Ulvales-Ulothrichales' delimited with ABGD and GMYC. Percent identity values were computed based on an alignment of $375 \mathrm{bp}$ of the tufA metabarcode. Maximum thresholds for molecular species classification/annotation are printed. P/ percent identity.

Additional file 11: Figure S4. OTU abundance profiles. OTUs underlying abundance patterns in Fig. 13. OTUs found in large read abundance across multiple microflora samples are color-coded in blue, green, orange and red. Other abundant OTUs are color-coded in white and those below $2 \%$ abundance in shades of grey. The OTUs low-level taxonomy and their percent match to the database are listed in the legend.

Additional file 12: Figure S5. Example of putative index tag jumping or tag error. Comparative read abundance for seven OTUs demultiplexed (i.e. assigned) to both FL02 and JP07. For illustration purpose, only OTUs with $>5 \%$ of mapped reads in either FL02 or JP07 are shown (these correspond to seven out of the 141 common OTUs, see Additional file 9: Figure S2). Note the drastic abundance disparity illustrative of or potential of false assignment via index tag jumping or tag error (e.g. OTU 511 representing $\ll 0.1 \%$ reads in FL02 and $10.4 \%$ of reads in JP07). By contrast, note the more balanced abundance of OTU 355,356 , and 506, which may reflect the true presence of common OTUs in both samples (i.e. pantropical OTUs).

Additional file 13: Figure S6. OTUs vs. clone identity and coverage. Comparison of all phototrophic clones obtained from JP07 with their matching OTUs. Note high identity of clones and OTUs based on $375 \mathrm{bp}$ with 0,1 or 2 base pair variation (100, 99.7 and $99.5 \%$ respectively). Also note that clone $\mathrm{H}$ and its corresponding OTU_364 share the highest coverage, although further cloning would be necessary to accurately assess congruence in sequence coverage between the two methods.

Additional file 14: Figure S7. tufA database and higher taxonomy overview. Unrooted RAxML tree comprising 556 'Heterotrophs'and 2141 'Phototrophs' tufA sequences aligned on 891 bp. 'Heterotrophs' include multiple phyla of the Eubacteria.'Phototrophs' include the prokaryotic eubacterial phylum Cyanophyta and several eukaryotic phyla, including the Archaeplastida (Chlorophyta, Glaucophyta and Rhodophyta) and secondary endosymbiotic phyla. 'Chloroplasts' include the Chlorophyta and the secondary endosymbiotic phyla Euglenophyta and Chlorarachniophyta. 'Rhodoplasts' include the Rhodophyta and the secondary endosymbiotic phyla of the Cryptophyta, Haptophyta and Ochrophyta.

\section{Abbreviations}

ABGD: automatic barcode gap discovery; bp: base pairs; $\mathrm{CaCO}_{3}$ : calcium carbonate; CCA: crustose coralline algae; EF-1a: elongation factor 1 alpha; ESS: effective sample size; $\mathrm{GeO}_{2}$ : germanium dioxide; GMYC: general mixed yule coalescence; ITS: internal transcribed spacer; LAF: University of Louisiana at Lafayette; LSU: large subunit; MCMC: Markov Chain Monte Carlo; NCBI: National Center for Biotechnology Information; $\mathrm{PCO}_{2}$ : partial pressure of carbon dioxide; PCR: polymerase chain reaction; PI: percent identity; OTU: Operational Taxonomic Unit; rbcL: RuBisCO large subunit; RDP: ribosomal database project; sp., spp.: species; tufA: elongation factor EF-Tu; tufB: elongation factor EF-Tu 2; UPA: universal plastid amplicon.

\section{Authors' contributions}

TS conducted field collections and culturing, summarized Genbank ${ }^{\circledR}$ content for tufA, and designed and tested novel degenerate primers. TS and WES generated reference Sanger data (including cloning data), performed bioinformatics and phylogenetics. TS conceptualized and drafted the manuscript. WES, SF and SS contributed to data interpretation, taxonomic expertise, collections and revision of the manuscript. SS hosted TS in Okinawa, Japan. All authors read and approved the final manuscript.

\section{Author details}

${ }^{1}$ Department of Biology, University of Louisiana at Lafayette, Lafayette, LA 70503, USA. ${ }^{2}$ Department of Marine Science, Biology and Chemistry, University of the Ryukyus, Nishihara, Okinawa 903-0213, Japan.

\section{Acknowledgements}

Field Sampling, Sanger sequencing, cloning, and next generation sequencing were primarily supported by the JSPS/CNRS Summer 2012 Fellowship \#SP12209 awarded to TS and from the "International Hub Project for Climate Change and Coral Reef/Island Dynamics of the University of the Ryukyus, Okinawa, Japan" Grant to SS, with additional contribution from the University of Louisiana at Lafayette Graduate Student Organization, Student Government Association and Center for Ecology and Environmental Technology (GrantsIn-Aid of Research) and a Link Foundation/Smithsonian Marine Station 2013 Fellowship awarded to TS. Sequencing of Florideophyceae and Phaeophyceae specimens were partly funded by the National Science Foundation (DEB0936855, DEB-1027107) and a Gulf of Mexico Research Initiative Coastal Water Consortium (GoMRI-I) Grant to SF. TS thanks Susan Brawley for the invitation to attend a bioinformatics training workshop offered during the Porphyra Algal Genomics' Research Collaboration Network (NSF 0741907), and EuroMarine/ EMBRC-France for further training provided during the 2015 Marine Ecological and Evolutionary Genomics in Roscoff, France. We are also thankful for help in the field from Yuhi Seto and Ooshi Hiraishi in the Ryukyus, and Olga Camacho, Natalia Arakaki and Joe Richards for miscellaneous specimens. We thank Alison Sherwood and Heather Spalding for specimens databased in Hawaii. We are grateful for constructive criticism provided by two anonymous reviewers on an early version of the manuscript.

\section{Competing interests}

The authors declare that they have no competing interests.

Received: 1 October 2015 Accepted: 27 February 2016

Published online: 10 March 2016

\section{References}

1. Abbott IA, Huisman JM. Marine green and brown algae of the Hawaiian Islands. Honolulu: Bishop Museum Press; 2004. p. 260.

2. Adams RI, Amend AS, Taylor JW, Bruns T. A unique signal distorts the perception of species richness and composition in high-throughput sequencing surveys of microbial communities: a case study of fungi in indoor dust. Microb Ecol. 2013;66:735-41.

3. Baldauf S, Palmer JD. Evolutionary transfer of the chloroplast tufA gene to the nucleus. Nature. 1990;344:262-5.

4. Bartram J, Carmichael WW, Chorus I, Jones G, Skulberg OM. Introduction. In: Chorus I, Bartram J, editors. Toxic cyanobacteria in water: a guide to their public health consequences, monitoring and management. London: WHO and E\&FN Spon; 1999. p. 1-14. 
5. Bendif EM, Probert I, Carmichael M, Romac S, Hagino K, de Vargas C. Genetic delineation between and within the widespread coccolithophore morpho-species Emiliania huxleyi and Gephyrocapsa oceanica (Haptophyta). J Phycol. 2014;50:140-8. doi:10.1111/jpy.12147.

6. Bláha L, Babica P, Maršálek B. Toxins produced in cyanobacterial water blooms - toxicity and risks. Interdiscip Toxicol. 2009;2:36-41. doi:10.2478/v10102-009-0006-2.

7. Bornet É, Flahault C. Sur quelques plantes vivantes dans le test calcaire des mollusques. Bull Soc Bot France. 1889;36:167-76.

8. Bouckaert R, Heled J, Kühnert D, Vaughan T, Wu C-H, et al. BEAST 2: a software platform for Bayesian evolutionary analysis. PLoS Comput Biol. 2014;10:e1003537. doi:10.1371/journal.pcbi.1003537.

9. Brodie J, Maggs CA, John DM. Green seaweeds of Britain and Ireland. London: British Phycological Society; 2007. p. 1-242.

10. Brown SDJ, Collins RA, Boyer S, Lefort MC, Malumbres-olarte J, Vink CJ, Cruickshank RH. Spider: an R package for the analysis of species identity and evolution, with particular reference to DNA barcoding. Mol Ecol Res. 2012;12:562-5.

11. Chaubey S, Kumar A, Singh D, Habib S. The apicoplast of Plasmodium falciparum is translationally active. Mol Microbiol. 2005;56:81-9.

12. Chazottes V, Le Campion-Alsumard T, Peyrot-Clausade M. Bioerosion rates on coral reef: interactions between macroborers, microborers and grazers (Moorea, French Polynesia). Palaeogeogr Palaeoclimat Palaeoecol. 1995:113:189-98.

13. Cole JR, Wang Q, Fish JA, Chai B, McGarrell DM, Sun Y, Brown CT, PorrasAlfaro A, Kuske CR, Tiedje JM. Ribosomal database project: data and tools for high throughput rRNA analysis. Nucl Acids Res. 2014;42(Database issue):D633-42. doi:10.1093/nar/gkt1244.

14. Cowart DA, Pinheiro M, Mouchel O, Maguer M, Grall J, Miné J, ArnaudHaond S. Metabarcoding Is powerful yet still blind: a comparative analysis of morphological and molecular surveys of seagrass communities. PLoS One. 2015. doi:10.1371/journal.pone.0117562.

15. Crooks GE, Hon G, Chandonia JM, Brenner SE. WebLogo: a sequence logo generator. Genome Res. 2004;14:1188-90.

16. Dawkins R, Krebs JR. Arms races between and within species. Proc R Soc Lond. 1979;205:489-511. doi:10.1098/rspb.1979.0081.

17. Decelle J, Romac S, Stern RF, Bendif EM, Zingone A, Audic S, Guiry MD, Guillou L, Tessier D, Le Gall L, Gourvil P, Dos Santos AL, Probert I, Vaulot $D$, de Vargas, Christen R. PhytoREF: a reference database of the plastidial $16 \mathrm{~S}$ rRNA gene of photosynthetic eukaryotes with curated taxonomy. Mol Ecol Resour. 2015;15(6):1435-45.

18. Degnan $\mathrm{PH}$, Ochman H. Illumina-based analysis of microbial community diversity. ISME J. 2012;6:183-94.

19. Delwiche CF, Kuhsel M, Palmer JD. Phylogenetic analysis of tufA sequences indicates a cyanobacterial origin of all plastids. Mol Phylog Evol. 1995:4:110-28.

20. Denonfoux J, Parisot N, DugatBony E, Biderre-Petit C, Boucher D, Morgavi DP, Paslier D, Peyretaillade E, Peyret P. Gene capture coupled to high-throughput sequencing as a strategy for targeted metagenome exploration. DNA Res. 2013;20(2):185-6.

21. DeSantis TZ, Hugenholtz P, Larsen N, Rojas M, Brodie EL, Keller K, Huber T, Dalevi D, Hu P, Andersen GL. Greengenes, a chimera-checked $16 \mathrm{~S}$ rRNA gene database and workbench compatible with ARB. Appl Env Microbiol. 2006;72:5069-72.

22. Dowle EJ, Pochon X, Banks JC, Shearer K, Wood SA. Targeted gene enrichment and high-throughput sequencing for environmental biomonitoring: a case study using freshwater macroinvertebrates. Mol Ecol Res. 2015. doi:10.1111/1755-0998.12488.

23. Draisma SGA, van Reine WFP, Sauvage T, Belton GS, Gurgel CFD, Lim $P E$, Phang SM. A re-assessment of the infra-generic classification of the gneus Caulerpa (Caulerpaceae, Chlorophyta) inferred from a timecalibrated molecular phylogeny. J Phycol. 2014;50:1020-34.

24. Edgar RC. MUSCLE: multiple sequence alignment with high accuracy and high throughput. Nucl Acids Res. 2004:32:1792-7.

25. Edgar RC. Search and clustering orders of magnitude faster than BLAST. Bioinformatics. 2010;26:2460-1.

26. Edgar RC. UPARSE: highly accurate OTU sequences from microbial amplicon reads. Nat Meth. 2013;10:996-8.

27. Eiler A, Drakare S, Bertilsson S, Pernthaler J, Peura S, Rofner C, Simek $\mathrm{K}$, Yang Y, Znachor P, Lindström ES. Unveiling distribution patterns of freshwater phytoplankton by a next generation sequencing based approach. PLoS One. 2013;8:e53516. doi:10.1371/journal.pone.0053516.

28. Esling P, Lejzerowicz F, Pawlowski J. Accurate multiplexing and filtering for high-throughput amplicon-sequencing. Nucleic Acids Res. 2015;43(5):2513-24. doi:10.1093/nar/gkv107

29. Famà P, Wysor B, Kooistra WHCF, Zuccarello GC. Molecular phylogeny of the genus Caulerpa (Caulerpales, Chlorophyta) inferred from chloroplast tufA gene. J Phycol. 2002;38:1040-50.

30. Felder DL, Thoma BP, Schmidt WE, Sauvage T, Self-Krayesky S, Chistoserdov A, Bracken-Grissom H, Fredericq S. Seaweeds and decapod crustaceans on Gulf deep banks after the Macondo Oil Spill. Bioscience. 2014;64:808-19.

31. Fine M, Loya Y. Endolithic algae: an alternative source of photoassimilates during coral bleaching. Proc R Soc Lond B Biol Sci. 2002;269:1205-10.

32. Fredericq S, Arakaki N, Camacho O, Gabriel D, Krayesky D, Self-Krayesky S, Rees G, Richards J, Sauvage T, Venera-Ponton T, Schmidt WE. A dynamic approach to the study of rhodoliths: a case study for the Northwestern Gulf of Mexico. Crypt Algol. 2014;35:77-98.

33. Fresnel J, Probert I. The ultrastructure and life cycle of the coastal coccolithophorid Ochrosphaera neapolitana (Prymnesiophyceae). Europ J Phycol. 2005;40:105-22.

34. Fučíková K, Leliaert F, Cooper ED, Škaloud P, D'hondt S, De Clerck O, et al. New phylogenetic hypotheses for the core Chlorophyta based on chloroplast sequence data. Front Ecol Evol. 2014;2:63.

35. Fujisawa T, Barraclough TC. Delimiting species using single-locus data and the generalized mixed Yule coalescent approach: a revised method and evaluation on simulated data sets. Syst Biol. 2013;62:707-24.

36. Glynn PW. Bioerosion and coral reef growth: a dynamic balance. In: Birkeland C, editor. Life and death of coral reefs. New York: Chapman and Hall; 1997. p. 68-95.

37. Guiry MD, Guiry GM. AlgaeBase. World-wide electronic publication. 2016; National University of Ireland, Galway. http://www.algaebase.org

38. Gutner-Hoch E, Fine M. Genotypic diversity and distribution of Ostreobium quekettii within scleractinian corals. Coral Reefs. 2011;30:643-50.

39. Händeler K, Wägele H, Wahrmund U, Rüdinger M, Knoop V. Slugs' last meals: kleptoplastidsin Sacoglossa (Opisthobranchia, Gastropoda): molecular identification of sequestered chloroplasts from different algal origins. Mol Ecol Res. 2010. doi:10.1111/j.1755-0998.2010.02853.x.

40. Hartman $H$, Favaretto $P$, Smith TF. The archaeal origins of the eukaryotic translational system. Archaea. 2006;2:1-9.

41. Hillis-Colinvaux L. Systematics of the siphonales. In: Irvine DEG, John DM, editors. Systematics of the green algae. London: Academic Press; 1984. p. 271-91.

42. Iwabe N, Kuma KI, Hasegawa M, Osawa S, Miyata T. Evolutionary relationship of archaebacteria, eubacteria, and eukaryotes inferred from phylogenetic trees of duplicated genes. Proc Natl Acad Sci USA. 1989:86:9355-9

43. Janouskovec J, Horák A, Oborník M, Lukes J, Keeling PJ. A common red algal origin of the apicomplexan, dinoflagellate, and heterokont plastids. Proc Natl Acad Sci USA. 2010;107:10949-54.

44. Jörger KM, Schrödl M. How to describe a cryptic species? Practical challenges of molecular taxonomy. Front Zool. 2013;10:59. doi:10.1186/1742-9994-10-59.

45. Ke D, Boissinot M, Huletsky A, Picard FJ, Frenette J, Ouellette M, Roy $\mathrm{PH}$, Bergeron MG. Evidence for horizontal gene transfer in evolution of elongation factor Tu in enterococci. J Bacteriol. 2000;182:6913-20.

46. Keeling PJ. Chromalveolates and the evolution of plastids by secondary endosymbiosis. J Eukaryot Microbiol. 2009;56:1-8.

47. Kelly MG, Whitton BA. The Trophic Diatom Index: a new index for monitoring eutrophication in rivers. J App Phycol. 1995;7:433-44.

48. Kitson JJN, Hahn C, Sands RJ, Straw NA, Evans DM, Lunt DH. Nested metabarcode tagging: a robust tool for studying species interactions in ecology and evolution. BioRxiv. 2016; doi:10.1101/035071

49. Köhler S, Delwiche CF, Denny PW, Tilney LG, Webster P, Wilson RJM, Palmer JD, Roos DS. A plastid of probable green algal origin in apicomplexan parasites. Science. 1997;275:1485-9.

50. Kraft GT. Algae of Australia: marine benthic algae of Lord Howe Island and the southern great barrier reef 1: green algae, 1. Collingwood: Australian Biological Resources Study and CSIRO Publishing; 2007. p. 365. 
51. Kumar PS, Brooker MR, Dowd SE, Camerlengo T. Target region selection is a critical determinant of community fingerprints generated by 16S Pyrosequencing. PLoS One. 2011;6:e20956. doi:10.1371/journal. pone.0020956.

52. Lapointe J, Braker-Gigras L. Translation mechanisms. Molecular biology intelligence unit. New York: Springer; 2014. p. 468

53. Lathe WC III, Bork P. Evolution of tuf genes: ancient duplication, differential loss and gene conversion. FEBS Lett. 2001;502:113-6.

54. Le Campion-Alsumard T, Golubic S, Hutchings P. Microbial endoliths in the skeletons of live and dead corals: Porites lobata (Moorea, French Polynesia). Mar Ecol Progr Ser. 1995;117:149-57.

55. Leliaert F, Lopez-Bautista JM. The chloroplast genomes of Bryopsis plumosa and Tydemania expeditiones (Bryopsidales, Chlorophyta): compact genomes and genes of bacterial origin. BMC Genom. 2015;16:204. doi:10.1186/s12864-015-1418-3.

56. Lemieux C, Otis C, Turmel M. A clade uniting the green algae Mesostigma viride and Chlorokybus atmophyticus represents the deepest branch of the Streptophyta in chloroplast genome-based phylogenies. BMC Biol. 2007;5:2. doi:10.1186/1741-7007-5-2.

57. Letunic I, Bork P. Interactive tree of life (itol): an online tool for phylogenetic tree display and annotation. Bioinformatics. 2007;21:127-8.

58. Linhart C, Shamir R. Degenerate primer design: theoretical analysis and the HYDEN program. Methods Mol Biol. 2007:402:221-44.

59. Logares R, Sunagawa S, Salazar G, Cornejo-Castillo FM, Ferrera I, Sarmento H, Hingamp P, Ogata H, de Vargas C, Lima-Mendez G, Raes J, Poulain J, Jaillon O, Wincker P, Kandels-Lewis S, Karsenti E, Bork P, Acinas SG. Metagenomic 16 S rDNA illumina tags are a powerful alternative to amplicon sequencing to explore diversity and structure of microbial communities. Env Microbiol. 2014;16:2659-71.

60. Lukas KJ. Taxonomy and ecology of the endolithic microflora of reef corals, with a review of the literature on endolithic microphytes. PhD Dissertation Univ Rhode Island, 1973; p. 159.

61. Lukas KJ. Two species of the Chlorophyte genus Ostreobium from skeletons of Atlantic and Caribbean reef corals. J Phycol. 1974;10:331-5.

62. Mahé $M$, Rognes T, Quince $C$, de Vargas C, Dunthorn M. Swarm: robust and fast clustering method for amplicon-based studies. PeerJ. 2014;2:e593. doi:10.7717/peerj.593.

63. Marin B, Nowack EC, Melkonian M. A plastid in the making: evidence for a second primary endosymbiosis. Protist. 2005;156:425-32.

64. Meinesz A. Sur le cycle de I'Halimeda tuna (Ellis et Solander) Lamouroux (Udoteaceae, Caulerpales). C R Acad Sci Paris. 1972;275:1363-5.

65. Mendoza MLZ, Sicheritz-Pontén T, Gilbert MT. Environmental genes and genomes: understanding the differences and challenges in the approaches and software for their analyses. Brief Bioinform. 2015. doi:10.1093/bib/bbv001.

66. Moniz MBJ, Guiry MD, Rindi F. TufA phylogeny and species boundaries in the green algal order Prasiolales (Trebouxiophyceae, Chlorophyta). Phycologia. 2014;53:396-406.

67. Montandon PE, Knuchel-Aegerter C, Stutz E. Euglena gracilis chloroplast DNA: the untranslated leader of tufA-ORF206 gene contains an intron. Nucl Acids Res. 1987;15:7809-22.

68. Odum HT, Odum EP. Trophic structure and productivity of a windward coral reef community on Eniwetok Atoll. Ecol Monographs. 1955;25:291-320.

69. Paradis E, Claude J, Strimmer K. APE: analyses of phylogenetics and evolution in R language. Bioinformatics. 2004;20:289-90.

70. Parker SP. Synopsis and classification of living organisms, New York: McGraw-Hill; 1982; 1:154.

71. Patel M, Gonzalez R, Halford C, Lewinski MA, Landaw EM, Churchill BM, Hake DA. Target-specific capture enhances sensitivity of electrochemical detection of bacterial pathogens. J Clin Microbiol. 2011;49:4293-6.

72. Perry CT, Murphy GN, Kench PS, Smithers SG, Edinger EB, Steneck RS, Mumby PJ. Caribbean-wide decline in carbonate production threatens coral reef growth. Nature Comm. 2013;4:1402. doi:10.1038/ ncomms2409.

73. Perry CT, Murphy GN, Kench PS, Edinger EN, Smithers SG, Steneck RS, Mumby PJ. Changing dynamics of Caribbean reef carbonate budgets: emergence of reef bioeroders as critical controls on present and future reef growth potential. Proc R Soc B. 2014;281:2014-8. doi:10.1098/ rspb.2014.2018
74. Price DC, Chan CX, Yoon HS, Yang EC, Qiu H, Weber AP, Schwacke R, Gross J, Blouin NA, Lane C, Reyes-Prieto A, Durnford DG, Neilson JA, Lang BF, Burger G, Steiner JM, Löffelhardt W, Meuser JE, Posewitz MC, Ball S, Arias MC, Henrissat B, Coutinho PM, Rensing SA, Symeonidi A, Doddapaneni H, Green BR, Rajah VD, Boore J, Bhattacharya D. Cyanophora paradoxa genome elucidates origin of photosynthesis in algae and plants. Science. 2012;335:843-7.

75. Provasoli L. Media and prospects for the cultivation of marine algae. In: Watanabe $\mathrm{H}$, Hattori A, editors. Culture and collection of Algae. Proc USA_Japan Conference. Jap Soc Plant Physiol Hakone. 1968; p. 63-75.

76. Puillandre N, Lambert A, Brouillet S, Achaz G. ABGD, automatic barcode gap discovery for primary species delimitation. Mol Ecol. 2012;21:186477. doi:10.1111/j.1365-294X.2011.05239.x.

77. Quast C, Pruesse E, Yilmaz P, Gerken J, Schweer T, Yarza P, Peplies J, Glöckner FO. The SILVA ribosomal RNA gene database project: improved data processing and web-based tools. Nucleic Acids Res. 2014;41:D590-6. doi:10.1093/nar/gks1219.

78. R Core Team. R: a language and environment for statistical computing. R foundation for statistical computing, Vienna: 2014. www.R-project. org.

79. Radtke G, Golubic S. Microborings in mollusc shells, Bay of Safaga, Egypt: morphometry and ichnology. Facies. 2005;51:118-34

80. Rambaut A, Drummond A. Tracer v1.6. 2007. Available from: http://tree. bio.ed.ac.uk/software/tracer.

81. Reyes-Nivia C, Diaz-Pulido G, Kline D, Guldberg OH, Dove S. Ocean acidification and warming scenarios increase microbioerosion of coral skeletons. Glob Chang Biol. 2013;19:1919-29.

82. Reyes-Nivia C, Diaz-Pulido G, Dove S. Relative roles of endolithic algae and carbonate chemistry variability in the skeletal dissolution of crustose coralline algae. Biogeosciences. 2014;11:4615-26.

83. Rodríguez-Ezpeleta N, Brinkmann H, Burey SC, Roure B, Burger G, Löffelhardt W, Bohnert HJ, Philippe H, Lang BF. Monophyly of primary photosynthetic eukaryotes: green plants, red algae, and glaucophytes. Curr Biol. 2005;15:1325-30.

84. Rohfritsch A, Payri C, Stiger V, Bonhomme F. Molecular and morphological relationships between two closely related species, Turbinaria ornata and T. conoides (Sargassaceae, Phaeophyceae). Biochem System Ecol. 2007;35:91-8.

85. Rohwer F, Seguritan $V$, Azam F, Knowlton N. Diversity and distribution of coral-associated bacteria. Mar Ecol Prog Ser. 2002;243:1-10.

86. Saunders GW, Kucera $H$. An evaluation of rbcL, tufA, UPA, LSU and ITS as DNA barcode markers from the marine green macroalgae. Cryptog Algol. 2010;31:487-528.

87. Sauvage T, Payri P, Draisma SGA, van Prud'homme Reine WF, Verbruggen $\mathrm{H}$, Sherwood A, Frederica S. Molecular diversity of the Caulerpa racemosa-Caulerpa peltata complex (Caulerpaceae, Bryopsidales) in New Caledonia, with new Australasian records for C. racemosa var. cylindracea. Phycologia. 2013;52:6-13.

88. Schmidt TSB, Matias Rodrigues JF, von Mering C. Limits to robustness and reproducibility in the demarcation of operational taxonomic units. Environ Microbiol. 2015;17:1689-706.

89. Schnell IB, Bohmann K, Gilbert MT. Tag jumps illuminated_reducing sequence-to-sample misidentifications in metabarcoding studies. Mo Ecol Res. 2015. doi:10.1111/1755-0998.12402

90. Sela S, Yogev D, Razin S, Bercovier H. Duplication of the tuf gene: a new insight into the phylogeny of eubacteria. J Bacteriol. 1989;171:581-4.

91. Sickel W, Ankenbrand MJ, Grimmer G, Holzschuh A, Härtel S, Lanzen J, Steffan-Dewenter I, Keller A. Increased efficiency in identifying mixed pollen samples by meta-barcoding with a dual-indexing approach. BMC Ecol. 2015;15:20. doi:10.1186/s12898-015-0051-y.

92. Simpson GG. Tempo and mode in evolution. New York: Columbia University Press; 1994.

93. Simpson GG. The major features of evolution. New York: Columbia University Press; 1953.

94. Sunagawa S, Wilson EC, Thaler M, Smith ML, Caruso C, Pringle JR, Weis VM, Medina M, Schwarz JA. Generation and analysis of transcriptomic resources for a model system on the rise: the sea anemone Aiptasia pallida and its dinoflagellate endosymbiont. BMC Genom. 2009;10:258. doi:10.1186/1471-2164-10-258.

95. Taanman JW. The mitochondrial genome: structure, transcription, translation and replication. Biochim Biophys Acta. 1999;1410:103-23. 
96. Taberlet P, Coissac E, Pompanon F, Brochmann C, Willerslev E. Towards next-generation biodiversity assessment using DNA metabarcoding. Mol Ecol. 2012;21:2045-50.

97. Tribollet A. Dissolution of dead corals by euendolithic microorganisms across the northern Great Barrier Reef (Australia). Microbial Ecol. 2008;5:569-80.

98. Tribollet A, Langdon C, Golubic S, Atkinson M. Endolithic microflora are major primary producers in dead carbonate substrates of Hawaiian coral reefs. J Phycol. 2006;42:292-303.

99. Tribollet A, Godinot C, Atkinson M, Langdon S. Effects of elevated pCO2 on dissolution of coral carbonates by microbial euendoliths. Global Biogeochem Cycl. 2009;23:GB3008. doi:10.1029/2008GB003286.

100. Tuohy TMF, Thompson S, Gesteland RF, Hughes D, Atkins JF. The role of EF-Tu and other translation components in determining translocation step size. Biochim Biophys Acta. 1990;1050:274-8.

101. Turmel M, Brouard JS, Gagnon C, Otis C, Lemieux C. Deep division in the Chlorophyceae (Chlorophyta) revealed by chloroplast phylogenomic analyses. J Phycol. 2008;44:739-50.

102. Verbruggen $H$, Ashworth M, LoDuca ST, Vlaeminck C, Cocquyt E, Sauvage T, Zechman FW, Littler DS, Littler MM, Leliaert F, De Clerck O. A multi-locus time-calibrated phylogeny of the siphonous green algae. Mol Phylogen Evol. 2009;50:642-53.

103. Verbruggen H, Vlaeminck C, Sauvage T, Sherwood AR, Leliaert F, De Clerck O. Phylogenetic analysis of Pseudochlorodesmis strains reveals cryptic diversity above the family level in the siphonous green algae (Bryopsidales, Chlorophyta). J Phycol. 2009;45:726-31.

104. Visco JA, Apothéloz-Perret-Gentil L, Cordonier A, Esling P, Pillet P, Pawlowski J. Environmental monitoring: inferring the diatom index from next-generation sequencing data. Environ Sci Technol. 2015;49:7597605. doi:10.1021/es506158m.

105. Wilson LJ, Weber XA, King TM, Fraser Cl. Extraction techniques for genomic analyses of macroalgae. In Seaweed Phylogeography 2016 The Netherlands: Springer; p. 363-386.

106. Zuccarello GC, West JA, Kikuchi N. Phylogenetic relationships within the Stylonematales (Stylenomatophyceae, Rhodophyta): biogeographic patterns do not apply to Stylonema alsidii. J Phycol. 2008;44:384-93. doi:10.1111/j.1529-8817.2008.00467.x.

\section{Submit your next manuscript to BioMed Central and we will help you at every step:}

- We accept pre-submission inquiries

- Our selector tool helps you to find the most relevant journal

- We provide round the clock customer support

- Convenient online submission

- Thorough peer review

- Inclusion in PubMed and all major indexing services

- Maximum visibility for your research

Submit your manuscript at www.biomedcentral.com/submit

() Biomed Central 